ARTICLE

\title{
Endurance exercise training-responsive miR-19b-3p improves skeletal muscle glucose metabolism
}

Julie Massart (10 1,8, Rasmus J. O. Sjögren 1,8, Brendan Egan (10 2,3, Christian Garde ${ }^{4}$, Magnus Lindgren1, Weifeng Gu ${ }^{5,7}$, Duarte M. S. Ferreira (1) ${ }^{6}$, Mutsumi Katayama ${ }^{2}$, Jorge L. Ruas (10) ${ }^{6}$, Romain Barrès (1) ${ }^{4}$, Donal J. O'Gorman ${ }^{3}$, Juleen R. Zierath (1) ${ }^{1,2,4} \&$ Anna Krook (iD ${ }^{2 凶}$

Skeletal muscle is a highly adaptable tissue and remodels in response to exercise training. Using short RNA sequencing, we determine the miRNA profile of skeletal muscle from healthy male volunteers before and after a 14-day aerobic exercise training regime. Among the exercise training-responsive miRNAs identified, miR-19b-3p was selected for further validation. Overexpression of miR-19b-3p in human skeletal muscle cells increases insulin signaling, glucose uptake, and maximal oxygen consumption, recapitulating the adaptive response to aerobic exercise training. Overexpression of miR-19b-3p in mouse flexor digitorum brevis muscle enhances contraction-induced glucose uptake, indicating that miR-19b-3p exerts control on exercise training-induced adaptations in skeletal muscle. Potential targets of miR-19b-3p that are reduced after aerobic exercise training include KIF13A, MAPK6, RNF11, and VPS37A. Amongst these, RNF11 silencing potentiates glucose uptake in human skeletal muscle cells. Collectively, we identify miR-19b-3p as an aerobic exercise training-induced miRNA that regulates skeletal muscle glucose metabolism.

\footnotetext{
${ }^{1}$ Department of Molecular Medicine and Surgery, Section of Integrative Physiology, Karolinska Institutet, Stockholm, Sweden. ${ }^{2}$ Department of Physiology and Pharmacology, Section of Integrative Physiology, Karolinska Institutet, Stockholm, Sweden. ${ }^{3}$ Department of Health \& Human Performance, Dublin City University, Dublin, Ireland. ${ }^{4}$ Novo Nordisk Foundation Center for Basic Metabolic Research, University of Copenhagen, Copenhagen, Denmark. ${ }^{5}$ University of Massachusetts Medical School, Worchester, MA, USA. ${ }^{6}$ Department of Physiology and Pharmacology, Section of Molecular and Cellular Exercise Physiology, Karolinska Institutet, Stockholm, Sweden. ${ }^{7}$ Present address: Department of Cell Biology and Neuroscience, University of California at Riverside, Riverside, CA, USA. ${ }^{8}$ These authors contributed equally: Julie Massart, Rasmus J. O. Sjögren. ${ }^{凶}$ email: anna.krook@ki.se
} 
T he benefits of exercise are pleiotropic, including systemic effects on cardiac function, energetics, and the vascular system, as well as intrinsic effects in working skeletal muscle including substrate metabolism and changes in contractile proteins and function ${ }^{1}$. Aerobic exercise training augments whole-body insulin sensitivity and improves glucose tolerance in healthy and insulin-resistant individuals ${ }^{2-4}$, thereby reducing the risk of developing type 2 diabetes 5 . While exercise training is recommended to alleviate insulin resistance in type 2 diabetic patients ${ }^{6}$, many of the molecular transducers of the effects of exercise in skeletal muscle remain to be identified.

Exercise-induced adaptations (i.e., increased mitochondrial density, altered substrate metabolism, or myofiber hypertrophy) in skeletal muscle are mediated by signaling events, pre- and posttranscriptional processes, regulation of translation, and ultimately, increased abundance and/or activity of proteins regulating these cellular processes ${ }^{7}$. Gene expression and protein abundance in skeletal muscle are robustly altered by aerobic exercise training 7,8 . These alterations are controlled by transcriptional networks involving numerous transcriptional regulators ${ }^{7}$. Transcription factors and epigenetic changes are candidate factors for such regulatory events. Mechanistic insight into the specific molecular and cellular events by which exercise enhances insulin sensitivity and preserves muscle mass may uncover novel therapeutic entry points to mimic or enhance exercise response in individuals unable to partake in training programs.

microRNAs (miRNAs) are a class of short, non-coding RNAs that regulate gene expression by reducing protein abundance of targeted genes ${ }^{9}$. miRNAs guide the RNA-induced silencing complex (RISC) to targeted mRNAs with $3^{\prime}$ UTR complementary sites, ultimately leading to reduced protein translation of miRNA targets. Aerobic exercise training remodels miRNA expression in skeletal muscle ${ }^{8,10}$. Several miRNAs are altered in response to acute aerobic exercise ${ }^{11}$, indicating the potential of miRNAdependent regulation of skeletal muscle exercise adaptation. However, the direct role of specific miRNAs in the regulation of gene expression and metabolism in skeletal muscle in response to exercise training remains unknown. Here we performed unbiased short RNA sequencing to determine the miRNA profile of human skeletal muscle and the adaptive response to aerobic exercise training. We hypothesized that miRNAs with altered expression following aerobic exercise training influence insulin sensitivity and energy substrate metabolism in skeletal muscle.

\section{Results}

Aerobic exercise training regulates multiple miRNAs in human skeletal muscle. The potential to repress target genes is considered to be mainly reserved for miRNAs with high cellular abundance $^{12}$. Thus, we performed short RNA sequencing to determine miRNAs expression levels in a subset of human skeletal muscle biopsies collected before and after an endurance exercise training program ( $n=3$ subjects). We identified 102 miRNAs with an expression level higher than 100 reads per million (RPM) in at least $50 \%$ of the biopsies (Supplementary Table 1). These 102 miRNAs represent skeletal muscle miRNAs with potential repressive activity either at rest, or in response to exercise training. Amongst these miRNAs, 93 had a mean expression above 100 RPM in biopsies collected before exercise training. An additional nine miRNAs had mean expression above 100 RPM in biopsies collected after exercise training. Several muscle-enriched miRNAs (myomiRs) including miR-1-3p, miR$133 \mathrm{a}-3 \mathrm{p}$, and miR-133b, were amongst the identified miRNAs with more than 10,000 RPM in biopsies collected before exercise training (Table 1). This analysis reveals the miRNA expression profile of human skeletal muscle.

\section{Table 1 Most abundant miRNAs in human skeletal muscle biopsies.}

MiRNA RPM

\begin{tabular}{lc}
\hline hsa-miR-1-3p & $208,397 \pm 61,354$ \\
hsa-miR-133a-3p & $37,577 \pm 16,582$ \\
hsa-let-7c-5p & $31,870 \pm 13,260$ \\
hsa-miR-133b & $31,238 \pm 15,654$ \\
hsa-let-7b-5p & $31,047 \pm 12,910$ \\
hsa-let-7a-5p & $29,562 \pm 12,453$ \\
hsa-let-7f-5p & $21,434 \pm 8186$ \\
hsa-miR-29c-3p & $19,759 \pm 5915$ \\
hsa-miR-451a & $18,272 \pm 7608$ \\
hsa-miR-206 & $12,371 \pm 1978$ \\
hsa-miR-23b-3p & $11,948 \pm 823$
\end{tabular}

Short RNA sequencing of human skeletal muscle biopsies obtained before exercise training was performed to determine miRNA abundance (reads per million (RPM) $>10,000$ ). Results are performed to determine miRNA
mean \pm SEM for three subjects.

Short RNA sequencing identified 12 miRNAs with differential expression in skeletal muscle after aerobic exercise training (Fig. 1A). In the absence of a specific probe for miR-378d, 11 of these 12 miRNAs were subjected to a secondary validation by RT-qPCR in skeletal muscle from eight volunteers, including the 3 subjects from the RNA sequencing analysis and 5 additional subjects undergoing the same aerobic exercise training program. RT-qPCR results were highly correlated with the short RNA sequencing data (Supplementary Fig. 1A). We found that 6 out of the 11 miRNAs showed altered abundance (Fig. 1B). The relatively less abundant myomiRs miR-1-5p and miR-133a$5 \mathrm{p}$ showed decreased expression following exercise training (Fig. 1B), consistent with a trend for decreased expression of other myomiRs (Supplementary Fig. 1B). We also identified increased expression of miR-19b-3p, miR-107, miR-223-3p, and miR-451a following exercise training (Fig. 1B). Skeletal muscle biopsies mainly consist of myocytes, but other cell types such as skeletal muscle progenitors (satellite cells), endothelial cells, and various circulating blood cells are present. To identify whether these miRNAs are expressed in myocytes, we determined their abundance in cultured human primary myotubes and compared these results with the abundance in skeletal muscle biopsies (Fig. 1C). We found miR-19b-3p and miR-107 are expressed to a similar extent in differentiated myotubes and biopsies, while miR-451a and miR-223-3p were undetectable in primary human myotubes (Fig. 1C). Most of the exercise training-regulated miRNAs identified in human skeletal muscle biopsies were also expressed in mouse soleus skeletal muscle even after removal of blood following cardiac perfusion (Supplementary Fig. 1C), with the exception of miR-451a, which showed expression to a similar degree as hemoglobin beta expression (Supplementary Fig. 1D), indicating that the primary source of miR-451a is of non-muscle origin. Given that miR-223-3p and miR-451a were not expressed in primary human skeletal muscle cells, the exercise-induced increase of these miRNAs is likely due to increased vascularization or infiltration of other cell types within the organ.

To gain further insight into the regulation of miR-19b-3p and miR-107 following exercise, we measured miR-19b-3p and miR107 expression at different timepoints throughout the training period. We found a time-dependent effect of the exercise training regimen to alter miR-19b-3p and miR-107 expression, with a significant increase after 14 days (Supplementary Fig. 1E, F). In a subset of five individuals undergoing the same aerobic exercise training program, we found increased expression of both miR19b-3p and miR-107 $16 \mathrm{~h}$ after the first exercise bout of the 
A

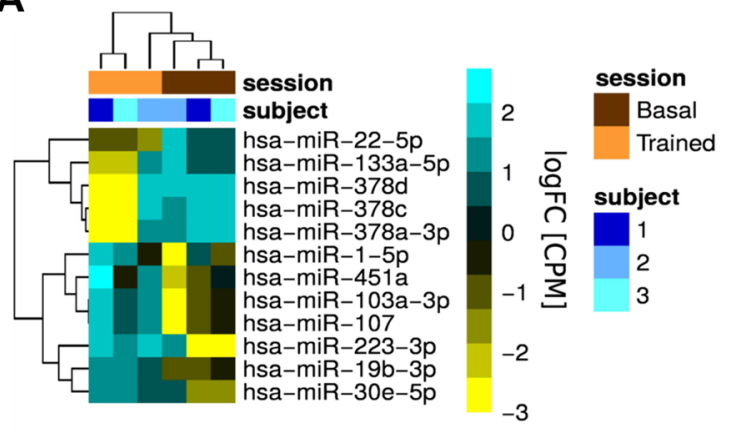

C

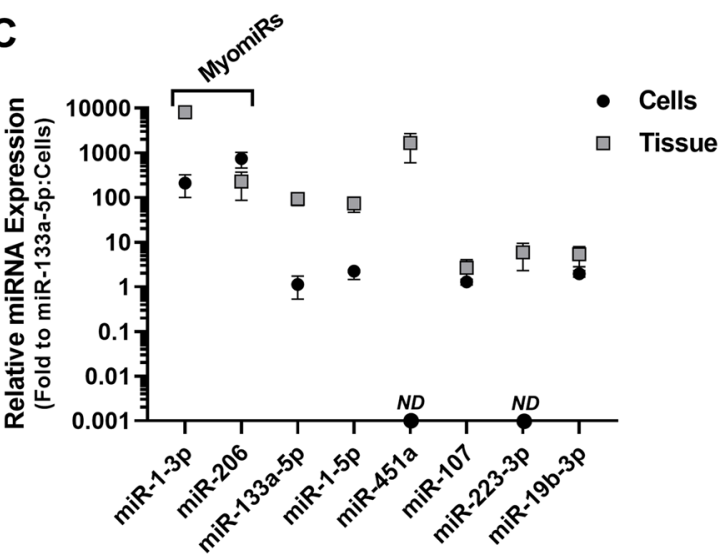

B

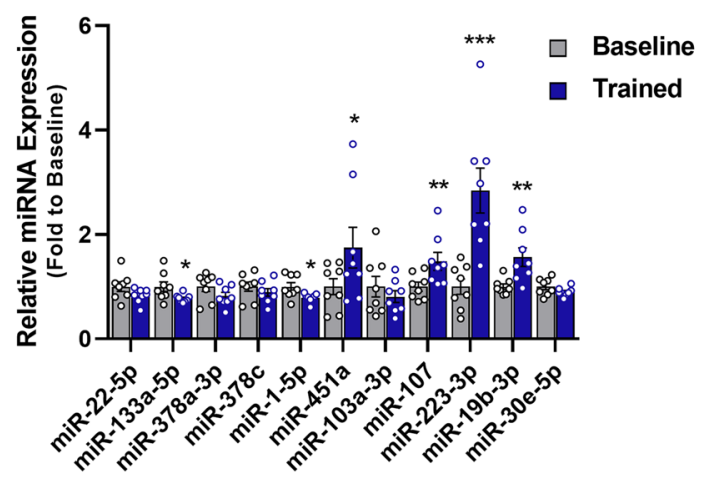

D

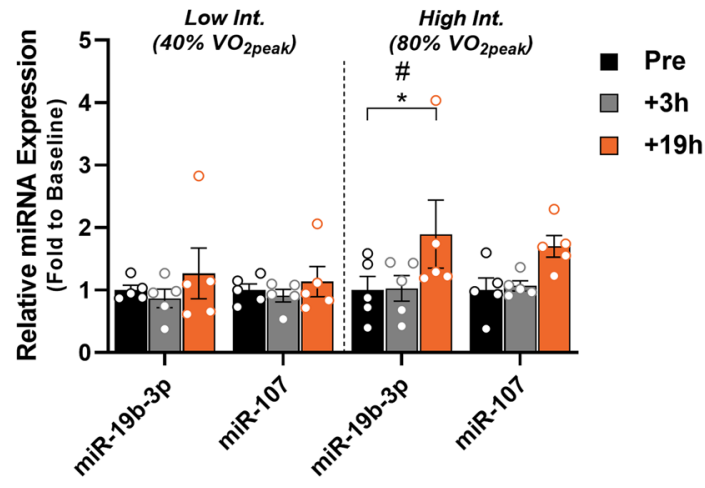

Fig. 1 Aerobic exercise training regulates multiple miRNAs in human skeletal muscle. Skeletal muscle biopsies were obtained from healthy volunteers before and after an aerobic exercise training program. A Altered miRNAs as determined by short RNA sequencing $(n=3$ individuals; FDR $<0.05)$. B RTqPCR determination of miRNA expression before (Baseline) and after 14-day aerobic exercise training (Trained) of miRNAs identified by short RNA sequencing shown in (A) ( $n=8$ individuals) ( $p=0.024$ for miR-133a-5p; $p=0.0236$ for miR-1-5p; $p=0.0224$ for miR-451a; $p=0.0029$ for miR-107; $p=0.0008$ for miR-223.3p; $p=0.0013$ for miR-19b-3p). C Relative miRNA expression in human skeletal muscle biopsies versus myotubes differentiated in vitro (data are mean \pm SD for $n=3$ cell culture independent samples and $n=8$ tissue independent samples). D miR-19b-3p and miR-107 expression determined by RT-qPCR in human skeletal muscle biopsies obtained at baseline, $3 \mathrm{~h}$, and $19 \mathrm{~h}$ after either low intensity $\left(40 \% \mathrm{VO}_{2 \text { peak }}\right)$ or high intensity $\left(80 \% \mathrm{VO}_{2 \text { peak }}\right)$ aerobic exercise $(p=0.0228$ for miR-19b-3p $19 \mathrm{~h}$ following high-intensity exercise; $n=5$ individuals). miRNA RT-qPCR data were normalized to miR-186-5p. Data are mean \pm SEM. \# Exercise effect (Friedman's test); ${ }^{\star} p<0.05,{ }^{\star \star} p<0.01,{ }^{\star \star *} p<0.001$ using two-tailed paired Student's ttest or Friedman's test followed by Dunn's multiple comparison test.

training regimen (Supplementary Fig. 1G, H). miR-19b-1-3p is part of the miR-17/92 cluster, which includes miR-20a-5p and miR-92a-3p. We therefore examined the expression of miR-20a$5 p$ and miR-92a-3p by RT-qPCR. miR-20a-5p was increased, while miR-92a-3p expression tended to increase (Supplementary Fig. 1I), suggesting transcriptional regulation of the cluster following exercise. To determine if exercise intensity regulates miR-19b-3p and miR-107 expression, we measured the levels of these miRNAs in skeletal muscle from subjects undergoing one bout of aerobic exercise at low $\left(40 \% \mathrm{VO}_{2 \text { peak }}\right)$ or high $(80 \%$ $\left.\mathrm{VO}_{2 \text { peak }}\right)$ intensity, at baseline, $3 \mathrm{~h}$, and $19 \mathrm{~h}$ after each respective exercise session. While expression of miR-19b-3p and miR-107 was unaffected by low-intensity exercise at every time point, expression of miR-19b-3p was increased at $19 \mathrm{~h}$, and miR-107 expression tended to be increased at $19 \mathrm{~h}$ after high-intensity exercise $(p=0.08)$ (Fig. 1D). Thus, transcriptional regulation of these two miRNAs occurs already at $19 \mathrm{~h}$ following moderate to high-intensity exercise. In addition, in mouse skeletal muscle, miR-19b-3p and miR-107 expression is higher in oxidative soleus muscle compared to glycolytic EDL muscle (Supplementary Fig. 1J). Since oxidative fibers are more recruited during endurance exercise compared to glycolytic fibers, these results are consistent with the increased expression following endurance exercise training. Thus, our analysis identifies miR-19b-3p and
miR-107 as two miRNAs that are responsive to endurance exercise in human skeletal muscle.

miR-19b-3p improves skeletal muscle glucose metabolism and insulin signaling. To determine the effects of miR-19b-3p and miR-107 on skeletal muscle glucose uptake, we overexpressed these miRNAs in differentiated human myotubes (Supplementary Fig. 2A, B). Basal and insulin-stimulated glucose uptake was increased following miR-19b-3p or miR-107 overexpression (Fig. 2A, B). In primary mouse skeletal muscle cells, miR-19b-3p overexpression enhanced insulin-stimulated glucose uptake (Fig. 2C and Supplementary Fig. 2C), while miR-107 overexpression was without effect (Fig. 2D and Supplementary Fig. 2D). Similarly, miR-19b-3p, but not miR-107, altered glucose uptake in C2C12 skeletal muscle cells (Supplementary Fig. 2E-H). To determine whether altered myotube differentiation accounts for changes in metabolism, we measured the expression of genes involved in skeletal muscle differentiation (Fig. 2E). In primary mouse skeletal muscle cells or in C2C12, miR-19b-3p, or miR-107 overexpression did not alter the expression of Desmin (DES) or the transcription factor governing muscle development Myogenin (MYOG) (Supplementary Fig. 2I, J). In human myotubes, miR$19 b-3 p$ overexpression decreased the expression of the proliferative marker Myogenic Factor 5 (MYF5) and increased the 

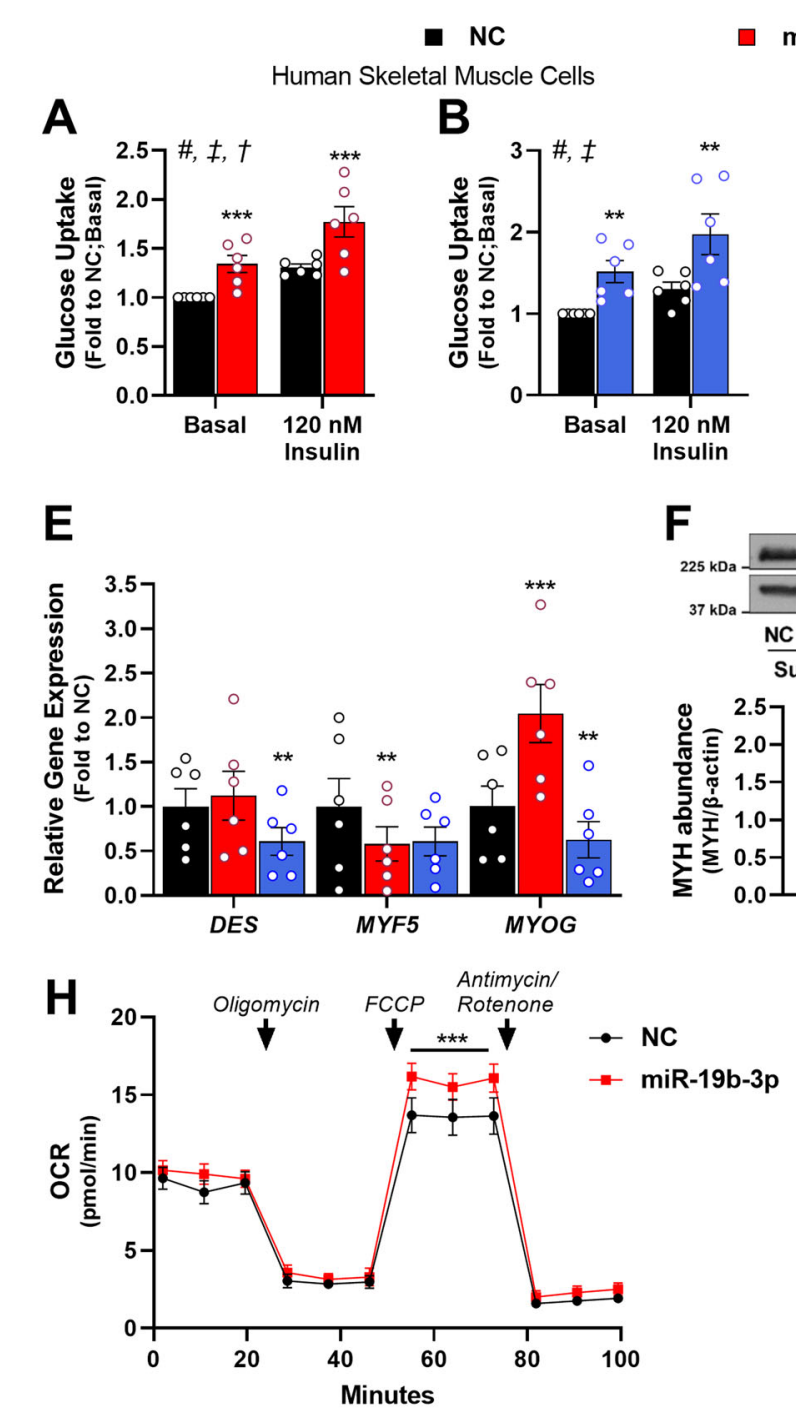

\section{K}

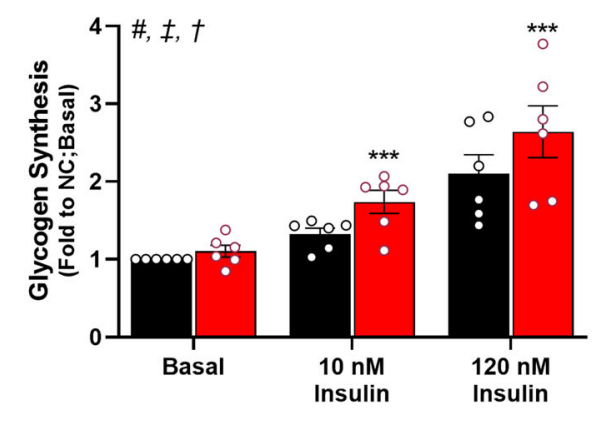

$\operatorname{miR}-19 b-3 p$

B

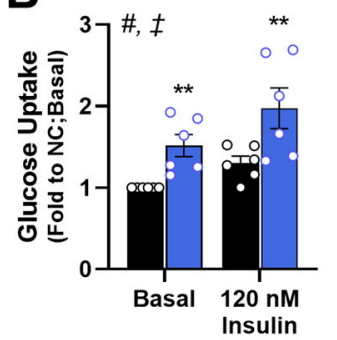

$\square$ miR-107

Mouse Skeletal Muscle Cells
C

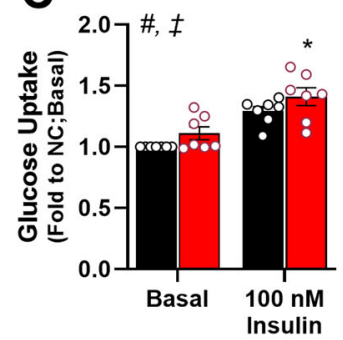

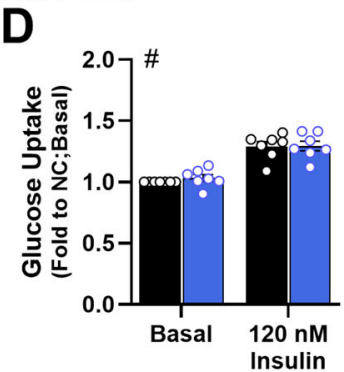
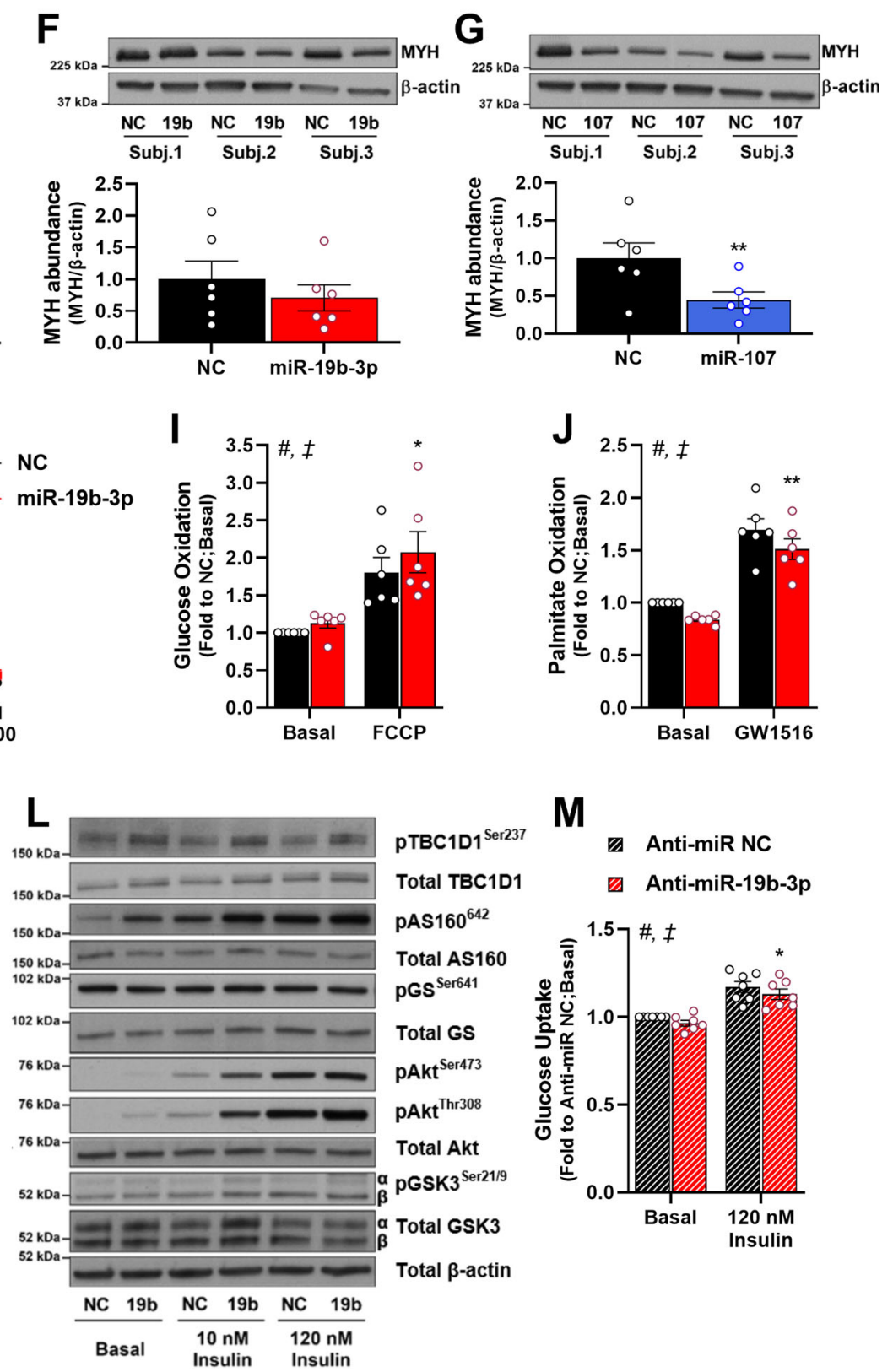

M
Anti-miR NC
Anti-miR-19b-3p

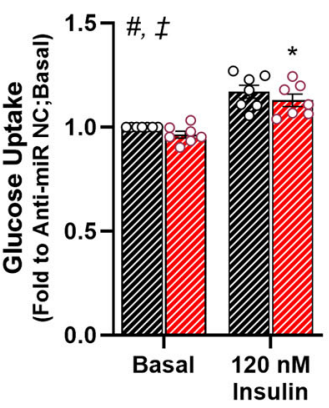

expression of MYOG (Fig. 2E), and was without effect on myosin heavy chain 1 and 2 (MYH1/2) protein abundance (Fig. 2F). Conversely, miR-107 overexpression decreased the expression of DES and MYOG and reduced MYH1/2 protein abundance in human skeletal muscle cells (Fig. 2E, G). These results indicate that miR-107 overexpression impairs human skeletal muscle cell differentiation. Given that the skeletal muscle differentiation status affects metabolism ${ }^{13}$, we further validated miR-19b-3p for a role on skeletal muscle metabolism.

Overexpression of miR-19b-3p improved maximal (FCCPstimulated) oxygen consumption rate (OCR) in human skeletal muscle cells (Fig. $2 \mathrm{H}$ ), indicative of increased reserve respiratory 
Fig. 2 miR-19b-3p improves glucose metabolism and insulin sensitivity in skeletal muscle. Myotubes were transfected with miR-19b-3p or miR-107 precursors or a negative control (NC) miRNA precursor. A, B Human skeletal muscle myotubes were incubated in the absence (Basal) or presence of insulin (120 nM) to determine the effect of $A)$ miR-19b-3p ( $p=0.0003$ for basal and $p=0.0001$ for insulin) and $\mathbf{B}$ miR-107 overexpression ( $p=0.0019$ for basal and $p=0.0014$ for insulin) on glucose uptake ( $n=6$ independent cell experiments). C, D Mouse skeletal muscle myotubes were incubated in the absence (Basal) or presence of insulin (100 nM) to determine effects of $\mathbf{C}$ miR-19b-3p ( $p=0.0307$ for insulin) and $\mathbf{D}$ miR-107 overexpression on glucose uptake ( $n=7$ independent cell experiments). E Expression of DES, MYF5, and MYOG was determined by RT-qPCR in human myotubes overexpressing either miR-19b-3p or miR-107 ( $n=6$ independent cell experiments). Data for human skeletal muscle cells were normalized to the geometrical mean of GUSB and TBP expression (miR-19b-3p: $p=0.0035$ for MYF5; $p=0.0008$ for MYOG; miR-107: $p=0.001$ for DES; $p=0.0057$ for MYOG). F, G Western blot analysis and quantification of Myosin Heavy Chain 1 and 2 (MYH) abundance in human myotubes overexpressing $\mathbf{F}$ miR-19b-3p and $\mathbf{G}$ miR-107 (miR107: $p=0.0067$ ) ( $n=6$ independent cell experiments). H Oxygen Consumption Rate (OCR) in human myotubes overexpressing miR-19b-3p ( $n=7$ independent cell experiments). Addition of chemical substrates are indicated by arrows. $p=0.0001$ following addition of FCCP. I Human myotubes were incubated in DMSO alone (Basal) or in the presence of $1 \mu \mathrm{M}$ of the mitochondrial uncoupler FCCP to determine the effects of miR-19b-3p overexpression on glucose oxidation ( $n=6$ independent cell experiments) ( $p=0.0241$ for FCCP). J Human myotubes were incubated in DMSO alone (Basal) or in the presence of $10 \mathrm{nM} \mathrm{GW1516} \mathrm{(PPAR \delta} \mathrm{agonist)} \mathrm{for} 96 \mathrm{~h}$ prior to determination of miR-19b-3p overexpression on palmitate oxidation ( $n=6$ independent cell experiments) ( $p=0.0026$ for basal and $p=0.0013$ for GW1516). $\mathbf{K}$ Human myotubes were incubated in the absence (Basal) or presence of insulin (10 or $120 \mathrm{nM}$ ) to determine the effect of miR-19b-3p overexpression on glucose incorporation into glycogen ( $n=6$ independent cell experiments) ( $p=0.0009$ for $10 \mathrm{nM}$ insulin and $p=0.0001$ for $120 \mathrm{nM}$ insulin). L Representative immunoblots of human myotubes incubated for 10 min in the absence (Basal) or presence of insulin (10 or $120 \mathrm{nM}$ ) to determine the effects of miR-19b-3p overexpression on protein phosphorylation and protein abundance. Data of immunoblot quantification are found in Table S2. M Human myotubes were transfected with a miR-19b-3p-specific inhibitor (Anti-miR-19b-3p) or a negative control inhibitor (Anti-miR NC) and incubated in the absence (Basal) or presence of insulin (120 nM) to determine the effects of miR-19b-3p inhibition on glucose uptake $(n=7)$. Data are mean \pm SEM. \# Treatment effect, $\uparrow$ miRNA effect, $\dagger$ Interaction; and ${ }^{\star} p<0.05,{ }^{\star \star} p<0.01,{ }^{\star \star *} p<0.001$ by paired Student's $t$-test or repeated measures 2-way ANOVA with Sidak's post hoc testing when appropriate.

capacity. Following miR-19b-3p overexpression, glucose oxidation was increased (Fig. 2I), while palmitate oxidation was decreased (Fig. 2J), indicating a shift towards glucose oxidation. Insulin sensitivity and responsiveness of glycogen synthesis were improved following miR-19b-3p overexpression (Fig. 2K). In primary mouse skeletal muscle cells, miR-19b-3p overexpression increased basal and insulin-stimulated glycogen synthesis (Supplementary Fig. 2K). In addition, miR-19b-3p increased phosphorylation of Akt at $\mathrm{Ser}^{473}$ and $\mathrm{Thr}^{308}$ at a submaximal insulin dose $(10 \mathrm{nM})$, with concomitant increased phosphorylation of downstream Akt targets such as AS160 ( $\mathrm{Thr}^{642}$ ) and GSK3a $\left(\mathrm{Ser}^{21}\right)$ (Fig. 2L and Supplementary Table 2). Overexpression of miR-19b-3p also increased phosphorylation of TBC1 Domain Family Member 1 (TBC1D1) at Ser ${ }^{237}$ with a concomitant increase in total TBC1D1 protein abundance (Fig. 2L and Supplementary Table 2). To determine the effects of endogenous miR-19b-3p on glucose metabolism, we utilized miRNA inhibitors in human myotubes to reduce miR-19b-3p availability (Supplementary Fig. 2L). Inhibition of miR-19b-3p reduced glucose uptake (Fig. $2 \mathrm{M}$ ), without affecting basal or insulin-stimulated glycogen synthesis (Supplementary Fig. 2M). Collectively, these results indicate that miR-19b-3p overexpression recapitulates exercise training-associated effects on skeletal muscle, including improved insulin sensitivity and glucose metabolism.

miR-19b-3p improves contraction-stimulated glucose transport in mouse skeletal muscle. To validate the role of miR-19b$3 p$ on glucose metabolism in vivo, we introduced a pri-miR-19b$3 \mathrm{p}$-encoding plasmid into mouse flexor digitorum brevis (FDB) muscle by electroporation. Seven days after electroporation the expression of miR-19b-3p was increased $\sim 2$-fold (Supplementary Fig. 3A). Overexpression of miR-19b-3p did not alter contractile properties in response to electrical pulse-stimulated contraction; relative force production over the time-course of contraction, maximal force production, or time to $50 \%$ fatigue were unaltered (Fig. 3A and Supplementary Fig. 3B, C). Interestingly, miR-19b$3 p$ overexpression was associated with an enhanced contractioninduced glucose uptake, whereas basal glucose transport was unaffected (Fig. 3B). We further assessed the effect of miR-19b-3p on glucose uptake in vivo with adenoviral vectors expressing $m i R$ $19 b-1$ gene. Viral infection resulted in $<2$-fold overexpression of mature miR-19b-3p as compared to control GFP muscles (Supplementary Fig. 3D). An overall effect of miR-19b-3p overexpression to increase glucose uptake in intact muscle was observed (Fig. 3C). Contraction-induced phosphorylation of TBC1D1 at Ser ${ }^{231}$ (human Ser ${ }^{237}$ ) was increased following miR$19 b-3 p$ overexpression, while Ser ${ }^{700}$ phosphorylation was unaltered (Fig. 3D, E). TBC1D1 is rapidly phosphorylated at Ser ${ }^{231}$ following skeletal muscle contraction by its upstream kinase AMP-activated protein kinase (AMPK) $)^{14}$, leading to increased translocation of GLUT4-containing vesicles and glucose transport. Intriguingly, overexpression of miR-19b-3p did not affect basal or contraction-induced phosphorylation of AMPK or its other downstream target acetyl-CoA carboxylase (ACC) (Fig. 3D, E). Although AMPK phosphorylation was unaffected, total abundance of AMPK a-subunits was increased by miR-19b-3p overexpression (Fig. 3D, E, G). In addition, miR-19b-3p overexpression increased abundance of mitochondrial complex subunits, indicating that oxidative phosphorylation is enhanced since abundance of succinate dehydrogenase complex iron sulfur subunit B (SDHB; complex II subunit) and mitochondrially encoded cytochrome c oxidase I (MTCO1; complex IV subunit) was increased (Fig. 3F, G). Abundance of other mitochondrial complex subunits (NDUFB8, UQCRC2, and ATP5A) and of GLUT4 was unaltered (Fig. 3F, G). Insulin-stimulated ( $0.36 \mathrm{nM})$ glucose transport and phosphorylation of Akt and GSK3a was unaltered by miR-19b-3p overexpression (Supplementary Fig. 3E-G). Thus, miR-19b-3p overexpression increases abundance of mitochondrial protein complexes and potentiates contraction-induced glucose uptake in intact skeletal muscle.

miR-19b-3p modulates expression of genes altered by endurance exercise training in human skeletal muscle. To identify the miR-19b-3p targets that control glucose metabolism, we first performed transcriptional profiling by microarray analysis of human skeletal muscle cells following miR-19b-3p overexpression. miR-19b-3p altered the expression of 679 genes (Fig. 4A and Supplementary Data 1), of which 208 were predicted targets of miR-19b-3p using the prediction target algorithms 
A

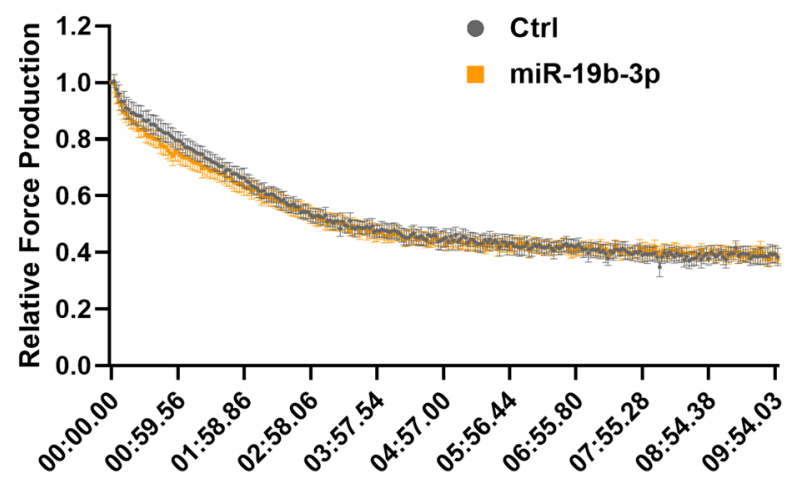

D

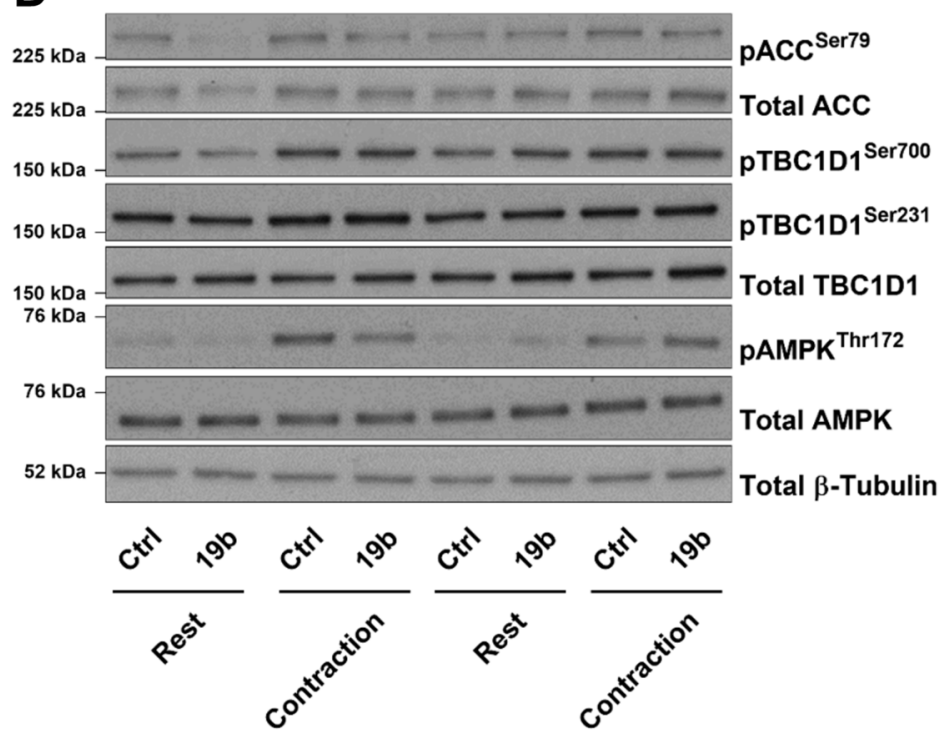

$\mathbf{F}$
B $\quad \rightarrow$ Ctrl $\quad \rightarrow$ miR-19b-3p

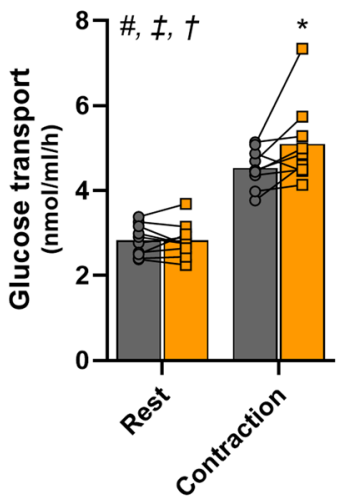

E
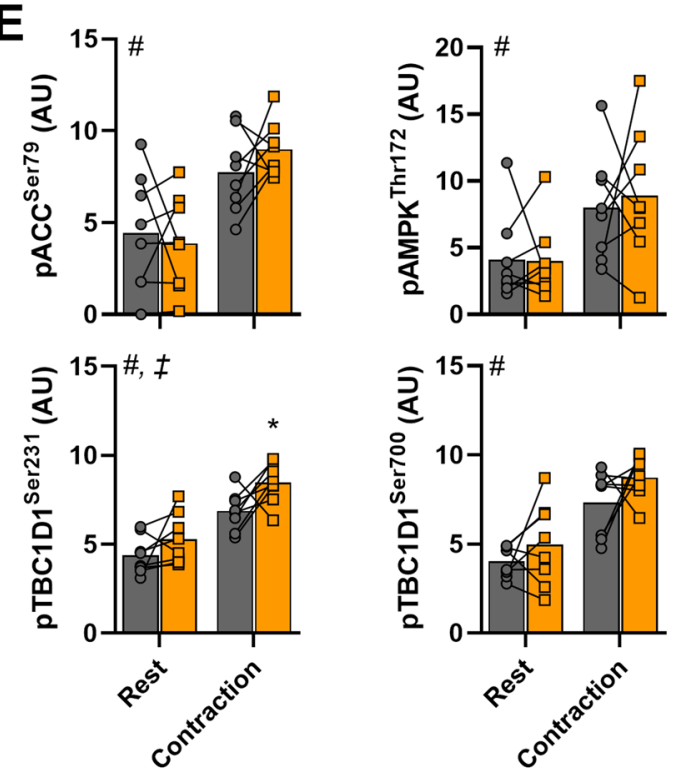

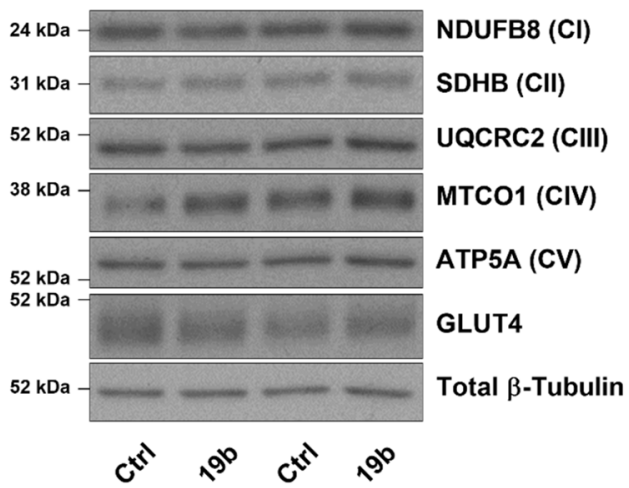
dicted target genes were downregulated. Gene Ontology (GO) enrichment analysis of differentially expressed genes in miR-19b$3 p$ overexpressing cells returned enrichment for genes related to extracellular matrix (ECM) organization (Supplementary Table 3). Since miR-19b-3p abundance was increased after aerobic exercise training, we aimed to discover putative exercise
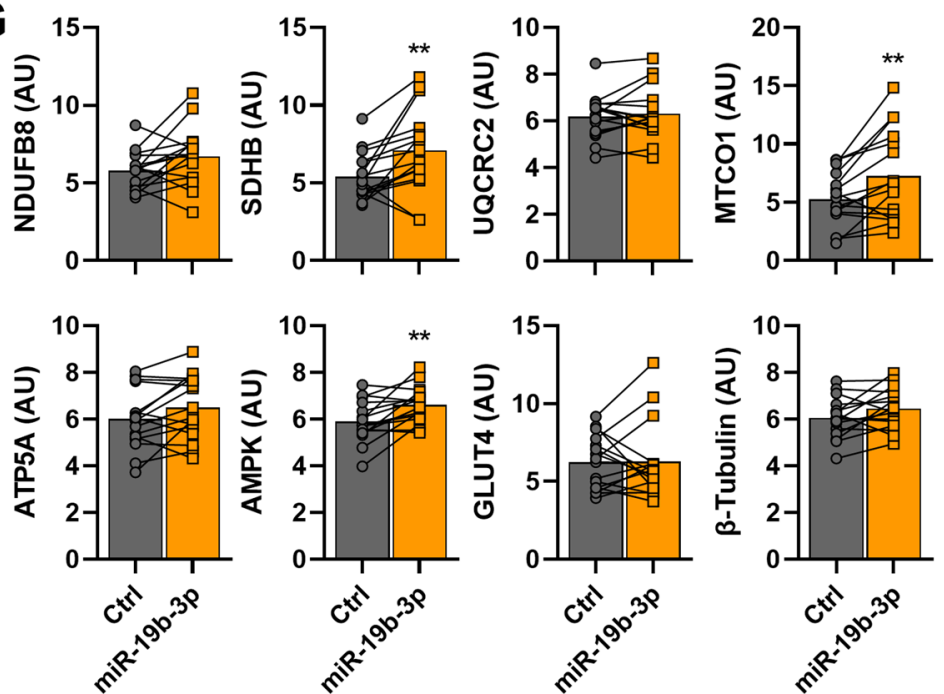
Fig. 3 miR-19b-3p improves contraction-stimulated glucose transport in mouse skeletal muscle. Mouse flexor digitorum brevis (FDB) skeletal muscle was electroporated with a control (Ctrl) or a pri-miR-19b-3p-encoding plasmid (miR-19b-3p) and 1 week later, was subjected to electrical pulse-stimulated contraction in vitro. A Relative force produced during each contraction measured over $10 \mathrm{~min}$ ( $n=9$ independent mouse muscles per construct). B In vitro glucose transport in FDB skeletal muscle electroporated with either control or pri-miR-19b-3p-encoding plasmid at rest or following electrical pulsestimulated contraction ( $p=0.0163$ ) ( $n=10$ for rest and $n=9$ for contraction independent mouse muscles per construct). C In vitro glucose transport in FDB skeletal muscle transduced with a control (Ad Ctrl) or mmu-miR-19b-1-expressing adenovirus (Ad miR-19b-1) at rest or following electrical pulsestimulated contraction ( $n=7$ independent mouse muscles). D Representative immunoblots of FDB skeletal muscle to determine the effects of miR-19b-3p overexpression on basal and contraction-induced protein phosphorylation or abundance. E Quantification of (D) for phosphorylation of ACC (Ser ${ }^{79}$ ), AMPK (Thr 172), and TBC1D1 (Ser231 and Ser ${ }^{700)}$ ( $\left(\right.$ TBC1D1 Ser ${ }^{231}: p=0.0479$ following contraction) ( $n=8$ independent mouse muscles). $\mathbf{F}$ Representative immunoblots of FDB skeletal muscle to determine the effects of miR-19b-3p overexpression on protein abundance. $\mathbf{G}$ Quantification of (F) for total abundance of NDUFB8, SDHB ( $p=0.0085)$, UQCRC2, MTCO1 $(p=0.005)$, ATP5A, AMPK ( $\alpha$-subunits; $p=0.0025)$, and GLUT4 ( $n=16$ independent mouse muscles). Data are mean. \# Contraction effect; $\uparrow$ miRNA effect; $\uparrow$ Interaction; and ${ }^{*} p<0.05$ and ${ }^{* \star} p<0.01$ using two-tailed paired Student's $t$-test or repeated measures two-way ANOVA with Sidak's post hoc testing when appropriate.

sedentary versus well-trained individuals ${ }^{15}$, or from healthy sedentary volunteers undergoing either a 6 -week ${ }^{8}$ or an 8 -week ${ }^{16}$ aerobic exercise-training program. Using a non-stringent $p$-value cutoff of $p<0.05$, we identified 460 unique genes altered by overexpression of miR-19b-3p and in at least one of the aerobic exercise-training studies (Fig. 4B). Of those, we identified $26 \mathrm{miR}$ $19 \mathrm{~b}-3 \mathrm{p}$ putative target genes overlapping at least two exercise training studies and having a similar fold change directionality following miRNA overexpression and after training (Fig. $4 \mathrm{~B}$ and Supplementary Table 4). We validated a subset of these predicted miR-19b-3p target genes by RT-qPCR following miR-19b-3p overexpression in human skeletal muscle cells, including CLIP4, HBP1, KIF13A, MAPK6, RNF11, VPS37A, ZBTB4, and ZDHHC7 (Fig. 4C). Similar results, with the exception of $Z b t b 4$, were observed in mouse skeletal muscle cells following miR-19b-3p overexpression (Supplementary Fig. 4A). Overexpression of miR$19 \mathrm{~b}-3 \mathrm{p}$ in mouse tibialis anterior (TA) muscle reduced the expression of Clip4, Kif13a, Mapk6, Rnf11, and Vps37a (Fig. 4D and Supplementary Fig. 4B). Seven days post-transduction, adenovirus-mediated mature miR-19b-3p overexpression in FDB muscle reduced the expression of 6 of the selected target genes, namely Clip4, Hbp1, Kif13a, Mapk6, Rnf11, and Vps37a (Supplementary Fig. 4C). Finally, we determined the expression of these eight miR-19b-3p predicted target genes in skeletal muscle biopsies of individuals undergoing aerobic exercise training. We found that aerobic exercise training reduced the expression of KIF13A, MAPK6, RNF11, and VPS37A (Fig. 4E). We found an inverse correlation between the training-induced fold change in miR-19b-3p expression and the expression of each of these four genes (Fig. 4F). Of interest, inhibition of miR-19b-3p in human skeletal muscle cells increased expression of RNF11 and VPS37A (Fig. 4G), further underscoring that miR-19b-3p directly regulates these genes. To determine the effects of miR-19b-3p target genes on glucose metabolism, we silenced KIF13A, MAPK6, RNF11, or VPS37A in human skeletal muscle cells (Fig. $4 \mathrm{H}$ and Supplementary Fig. 4D). Silencing of RNF11 increased basal and insulin-stimulated glucose uptake, mimicking miR-19b-3p overexpression (Fig. 4I). RNF11 silencing did not alter basal or insulin-stimulated glycogen synthesis (Fig. 4J). Conversely, silencing the other genes did not alter glucose uptake or glycogen synthesis in human skeletal muscle cells (Supplementary Fig. 4E-J). Thus, while RNF11 may contribute to the miR-19b$3 \mathrm{p}$-induced alterations of glucose uptake, it alone is unlikely to explain the full spectra of metabolic changes induced by miR19b-3p.

\section{Discussion}

miRNAs are post-transcriptional regulators involved in fine-tuning several biological processes. Using an unbiased short RNA sequencing approach, we describe the miRNA profile of human skeletal muscle and identify aerobic exercise-responsive miRNAs in healthy young volunteers. We show aerobic exercise training alters the expression of several miRNAs in skeletal muscle, including miR-19b-3p. Overexpression of miR-19b-3p in cultured human skeletal muscle cells increases glucose uptake and oxidation. Furthermore, miR-19b-3p overexpression increases canonical insulin signaling at the level of Akt and AS160, consistent with enhanced insulin sensitivity. Furthermore, our efforts to mimic the profile of exercise-trained muscle through overexpression of miR-19b-3p in intact mouse skeletal muscle, provided evidence that miR-19b-3p controls contraction-mediated glucose uptake. Transcriptomic analysis revealed networks regulated by miR-19b-3p involved in skeletal muscle remodeling and metabolism. miR-19b-3p regulates several targets found to be altered in response to aerobic exercise training, including KIF13A, MAPK6, or RNF11. Silencing of RNF11, a protein previously unstudied in skeletal muscle, resulted in an increased glucose uptake. Thus, RNF11 may partially account for exercise- and miR-19b-3p-mediated increase in skeletal muscle glucose metabolism.

Exercise training-responsive miRNAs have been explored by both unbiased microarray and RT-qPCR analysis of selected candidate miRNAs ${ }^{8,10}$. These approaches depend on prior sequence knowledge. In contrast, RNA sequencing requires no prior sequence knowledge of constituent RNA. The RNA sequencing data reported in this study provides an unbiased miRNA expression profile of untrained and aerobic exercise-trained human skeletal muscle. Consistent with previous studies ${ }^{8,10}$, we found that aerobic exercise training reduced the expression of several members of the myomiR family, including miR-133a-5p, miR-1-5p, and miR-133b. Trainingspecific responses on myomiR expression have been reported. Aerobic exercise training reduced myomiR expression, while acute aerobic exercise increased miR-1 and miR-133 expression ${ }^{10}$. In contrast, resistance exercise training had no effect ${ }^{17}$. The trainingspecific alterations of myomiR expression may arise from remodeling processes that depend on intensity, duration, and type of exercise.

Skeletal muscle is a complex tissue, and the effects of exercisetraining are not solely restricted to myocytes. We identified four miRNAs that were increased in skeletal muscle after exercise training; miR-19b-3p, miR-107, miR-223-3p, and miR-451a. miR$19 \mathrm{~b}-3 \mathrm{p}$ and miR-107 are expressed in myocytes, whereas miR-223$3 \mathrm{p}$ and miR-451a are likely expressed in cells of non-muscle origin. miR-451a is enriched in erythrocytes, with relatively low abundance in skeletal muscle ${ }^{18}$, and circulating levels of miR-223-3p are increased following endurance exercise, with expression levels linked to the inflammatory response associated with exercise ${ }^{19,20}$. Besides acting intracellularly, miRNAs can be secreted to neighboring cells or into the circulation, and therefore they may be involved in cell-to-cell signaling and communication ${ }^{21}$. Thus, miR-223-3p and miR-451a may act in a paracrine manner in 


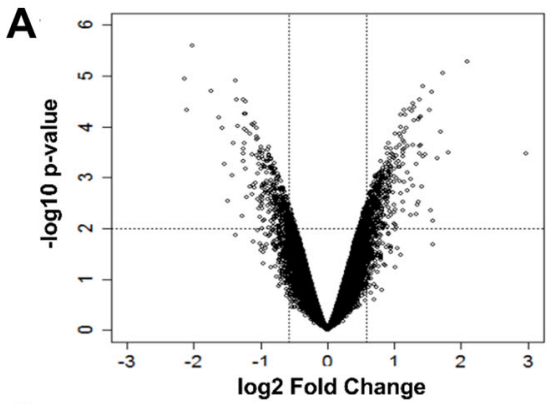

C
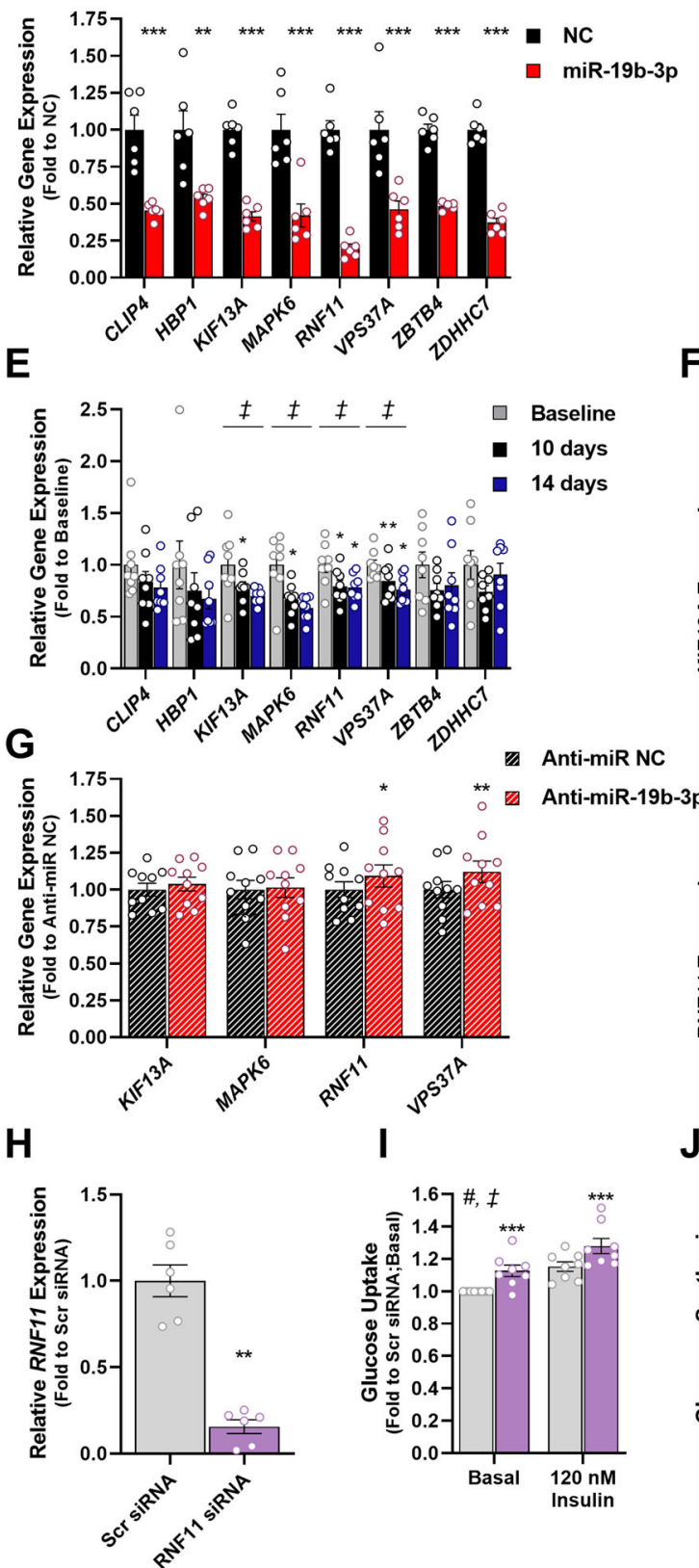

skeletal muscle in response to exercise training. Skeletal muscle expression of miR-107 is increased following acute treadmill running ${ }^{22}$ and decreased by hind-limb suspension ${ }^{23}$ in mouse models. We report that miR-107 alters differentiation of human myotubes, consistent with findings of reduced myogenesis in bovine skeletal muscle cells ${ }^{24}$. Thus, miR-107 appears to play a role in skeletal muscle remodeling. Given that the differentiation state

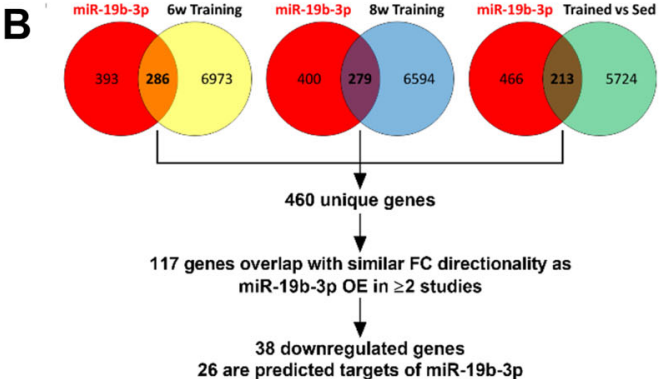

D
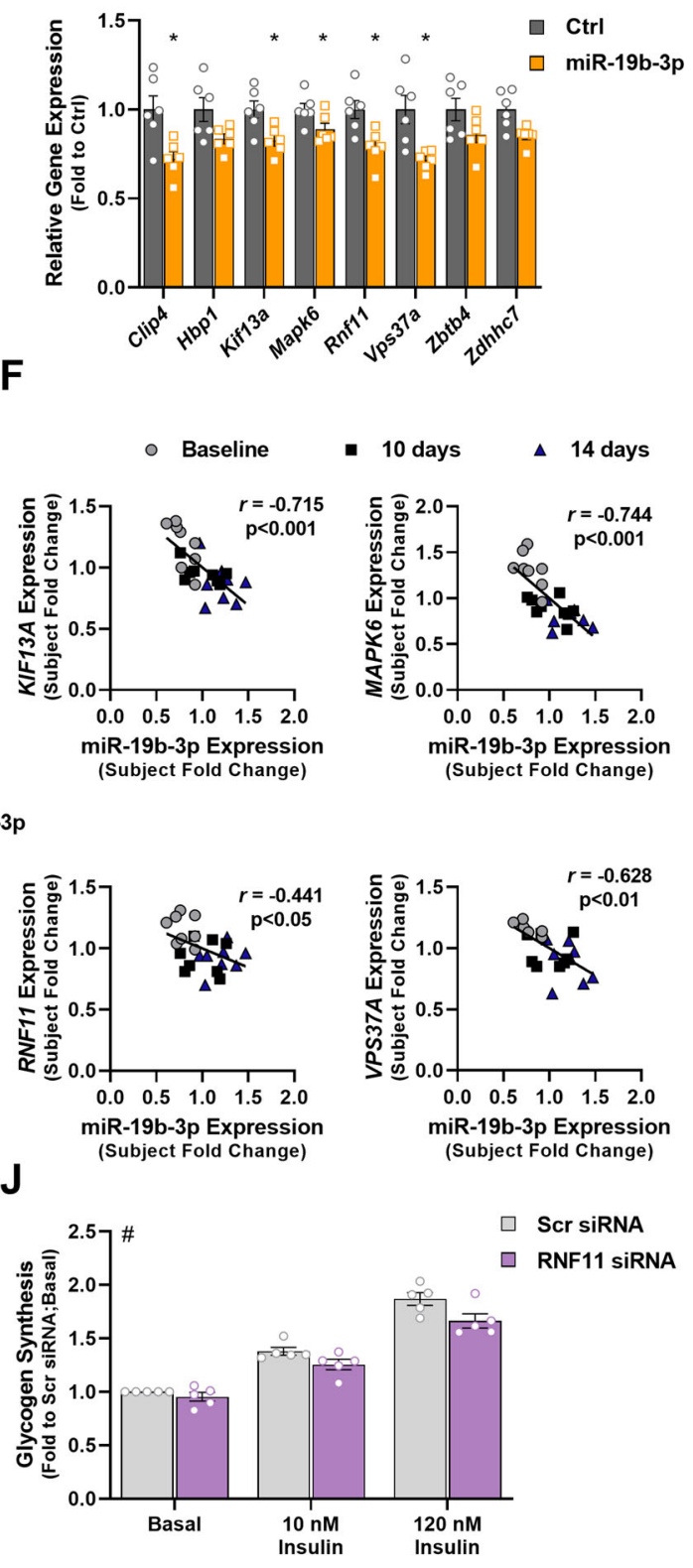

has a profound effect on metabolism ${ }^{13}$, we deprioritized further studies on miR-107. Consequently, we selected the exerciseregulated miR-19b-3p for further validation to gain insight into mechanisms by which aerobic exercise training alters insulin sensitivity and metabolism in skeletal muscle.

The molecular networks contributing to the adaptation to exercise in human skeletal muscle are not fully identified. While 
Fig. 4 miR-19b-3p modulates expression of genes altered by endurance exercise training in human skeletal muscle. A $\vee o l c a n o$ plot showing changes in gene expression determined by microarray analysis of myotubes overexpressing miR-19b-3p. Dashed lines indicate value cut-off at $p<0.01$ and fold change of at least $\pm 50 \%$ ( $n=4$ independent cell culture experiments). B Overview of workflow to identify genes with altered expression profiles following miR19b-3p overexpression in human skeletal muscle cells and following aerobic exercise training (analysis of publicly available data8,15,16). C Expression of miR-19b-3p predicted target genes CLIP4 $(p=0.0001)$, HBP1 $(p=0.0032)$, KIF13A $(p=0.0001)$, MAPK6 $(p=0.0007), R N F 11(p=0.0001)$, VPS37A $(p=0.0004), Z B T B 4(p=0.0001)$, and ZDHHC7 $(p=0.0001)$ as determined by RT-qPCR following negative control (NC) or miR-19b-3p overexpression in human skeletal muscle cells ( $n=6$ independent cell culture experiments). D Effect of miR-19b-3p overexpression in mouse tibialis anterior skeletal muscle on expression of Clip4 ( $p=0.0275), H b p 1, K i f 13 a(p=0.0388)$, Mapk6 $(p=0.0116), \operatorname{Rnf11}(p=0.034)$, Vps37a $(p=0.0206), Z b t b 4$, and Zdhhc7 as determined by RT-qPCR ( $n=6$ independent mouse samples). E Gene expression of CLIP4, HBP1, KIF13A ( $p=0.0497$ at day 10), MAPK6 ( $p=0.0143$ at day 10 and $p=0.0031$ at day 14$), R N F 11(p=0.038$ at day 10 and $p=0.017$ at day 14$)$, VPS37A $(p=0.0051$ at day 10 and $p=0.0222$ at day 14$), Z B T B 4$, and ZDHHC7 as determined by RT-qPCR in human skeletal muscle biopsies collected before (Baseline) and after 10 or 14 days of endurance exercise training $(n=8$ individuals). F Pearson correlation of fold changes in miR-19b-3p expression and fold change of KIF13A $(p=0.0001), M A P K 6(p=0.0001), R N F 11$ ( $p=0.0312$ ), or VPS37A ( $p=0.001)$ expression in human skeletal muscle biopsies collected prior to (Baseline) and after 10 or 14 days of endurance exercise training ( $n=24$ biopsies). G Human myotubes were transfected with a miR-19b-3p-specific inhibitor (Anti-miR-19b-3p) or a negative control inhibitor (Anti-miR NC) and gene expression of KIF13A, MAPK6, RNF11 $(p=0.024)$, and VPS37A $(p=0.0034)$ was determined by RT-qPCR $(n=10$ independent cell culture experiments). H Human skeletal muscle myotubes were transfected with a scramble siRNA sequence (Scr siRNA) or a RNF11 siRNA and gene expression of RNF11 was determined by RT-qPCR ( $p=0018, n=6$ independent cell culture experiments). I Human skeletal muscle myotubes were incubated in the absence (Basal, $p=0.0004$ ) or presence of insulin ( $120 \mathrm{nM}, p=0.0008$ ) to determine the effect of RNF11 silencing on glucose uptake ( $n=8$ independent cell culture experiments). J Glycogen synthesis rate was determined in human skeletal muscle cells in the absence (Basal) or presence of insulin (10 or $120 \mathrm{nM}$ ) following RNF11 silencing ( $n=5$ independent cell culture experiments). Data are mean \pm SEM. RT-qPCR data in human skeletal muscle biopsies and cells were normalized to the geometrical mean of GUSB and TBP expression. For mouse TA muscle, gene expression was normalized to Tbp expression. \# Insulin effect; $\$$ Endurance exercise training effect; and ${ }^{\star} p<0.05,{ }^{\star \star} p<0.01,{ }^{\star \star *} p<0.001$ by two-tailed paired Student's $t$-test, repeated measures one-way ANOVA with Dunnett's post hoc testing, or repeated measures two-way ANOVA with Sidak's post hoc testing when appropriate.

specific transcription factors and co-factors, such as NR4As, PPARs, or PGC-1 $\alpha$ are involved in the response to a single bout of exercise, there is a coordinated transcriptional response to exercise which is both time- and intensity-dependent ${ }^{7}$. Some genes are regulated during and directly after exercise (for example TFAM $)^{25}$ and others are altered in the recovery $(\sim 3 \mathrm{~h})$ and adaptive $(>12 \mathrm{~h}$ ) phases following exercise (such as PGCla and PPAR $\delta)^{25}$. We provide evidence that miR-19b-3p expression is induced $19 \mathrm{~h}$ post-exercise cessation and that elevation of miR19b-3p transcripts requires moderate- to high-intensity exertion. Since other members of the miR-17/92 cluster were also increased, it is likely that elevated miR-19b-3p levels after exercise training is driven by transcriptional regulation, and not regulation of miR-19b-3p stability. Myc is a candidate transcription factor regulating miR-19b-3p transcription as it is induced within 30 min post-exercise ${ }^{26}$ and can directly bind to the promoter of the miR-17/92 cluster and initiate transcription ${ }^{27}$. However, we cannot exclude that miR-19b-3p is also independently regulated by other post-transcriptional mechanisms, such as acetylated AGO2 favoring the production of mature miR-19b-3p from the pre-miRNA ${ }^{28}$. Overall, we show that miR-19b-3p expression is increased in the adaptive phase $(>12 \mathrm{~h})$ after exercise training and may play a role in directing muscle remodeling following moderate to high-intensity exercise. The increase in miR-19b-3p is maintained during 14 days of endurance training.

Regular exercise improves insulin sensitivity, glucose metabolism, and mitochondrial biogenesis in skeletal muscle ${ }^{7}$, but the role of miRNAs in orchestrating these changes remains largely unknown. Thus, we assessed whether miR-19b-3p, an exercise training-induced miRNA, affects skeletal muscle metabolism. In cultured human skeletal muscle cells, inhibition of endogenous miR-19b-3p reduced insulin-stimulated glucose uptake, and overexpression of miR-19b-3p enhanced glucose uptake. Likewise, in mouse skeletal muscle, a modest overexpression of miR19b-3p enhanced contraction-induced glucose transport, similar to exercise training ${ }^{29}$. While the effect size of the increase in miR$19 b-3 p$ enhanced glucose uptake was modest, given that skeletal muscle mass represents up to $50 \%$ of total body mass, even small enhancements are likely to significantly impact whole-body glucose homeostasis. The duration and magnitude in miR-19b-3p overexpression achieved in mouse muscle were similar to that noted in response to exercise training in the young healthy men, supporting a physiological role of miR-19b-3p in regulation of glucose transport. These improvements in glucose uptake may be linked to changes in signal transduction. We found TBC1D1Ser231 phosphorylation was increased by miR-19b-3p overexpression, consistent with the response of this signal transducer to acute exercise $^{30}$. We further determined the metabolic fate of glucose in response to miR-19b-3p overexpression in myotubes, and noted increased oxidation and incorporation into glycogen. Increased glucose transport and glycogen synthesis are hallmarks of exercise training ${ }^{31}$, however, increased lipid oxidation is another mechanism by which exercise training improves metabolism ${ }^{32}$. We found miR-19b-3p overexpressing cells preferentially oxidized glucose over lipids, which may reflect the predominantly glycolytic cell culture conditions, rather than the substrates available to the working muscle during exercise. In intact mouse muscle, miR-19b-3p overexpression increased the abundance of mitochondrial proteins, consistent with effects of exercise training on the mitochondrial proteome ${ }^{33}$. Overall our results provide evidence that miR-19b-3p recapitulates part of the effects of exercise training on mitochondrial markers and glucose metabolism.

The identification of target genes mediating the functional effects of miRNAs is challenging. We applied a transcriptomic approach to determine the effects of miR-19b-3p overexpression on gene expression in human skeletal muscle cells. We found that miR-19b-3p overexpression in myotubes regulates genes involved in extracellular matrix (ECM) remodeling, a process implicated in exercise-training-related adaptations in skeletal muscle, with expression of ECM genes linked to the magnitude of the aerobic exercise training response $\mathrm{e}^{34}$. This suggests that in addition to the regulation of glucose metabolism, miR-19b-3p may also control processes involved in maintaining the structural integrity and functional properties of skeletal muscle ${ }^{35}$. However, we did not observe marked change of force production in intact mouse 
muscle following a modest overexpression of miR-19b-3p. Of note, flexor digitorum brevis muscle is more fatigue-resistant than other muscle types ${ }^{36}$, thus we cannot exclude the possibility that the electrical stimulation protocol (strength versus endurance) or type of muscle studied (fatigue prone versus fatigue resistant) influenced these results.

To ascertain whether the supraphysiological overexpression of miR-19b-3p drives non-specific effects, including alterations in gene expression measured by microarray, we compared the myotube transcriptomic profile with that of endurance-trained skeletal muscle $8,15,16$. We identified 26 predicted targets downregulated both by miR-19b-3p overexpression and aerobic exercise training. We confirmed that several of these predicted targets, including KIF13A, MAPK6, RNF11, and VPS37A, were also regulated by exercise training in our cohort. RNF11 silencing enhanced basal and insulin-stimulated glucose uptake, similar to miR-19b-3p overexpression. RNF11 is a miR-19b-3p validated target by luciferase assay 37 , and is an essential component of ubiquitin-editing, implicated in NF- $\mathrm{BB}$ activation and inflammation ${ }^{38}$. RNF11 has a 14-3-3 binding sequence and an Akt phosphorylation site ${ }^{39}$, potentially linking it to functional events downstream of Akt including growth and glucose metabolism. Thus, the effects of miR-19b-3p on metabolism may be partially mediated by RNF11.

Using short RNA sequencing we describe the human skeletal muscle miRNA profile and identify aerobic exercise trainingresponsive miRNAs. We validate miR-19b-3p as an aerobic exercise training-induced miRNA that regulates skeletal muscle insulin sensitivity and glucose metabolism. Of the predicted miR19b-3p targets altered in skeletal muscle after exercise training, RNF11 regulates glucose uptake. In conclusion, miR-19b-3p contributes to exercise training-induced improvements in skeletal muscle partly via RNF11.

\section{Methods}

Exercise study cohorts. All participants provided written informed consent and the research ethical committee at Dublin City University approved the study protocols and experimental procedures were conducted according to the Declaration of Helsinki. Two separate cohorts were used.

Human participants for exercise training study. Eight healthy, sedentary men ( $23 \pm 2$ years of age) volunteered to participate in a 14-day consecutive training program as described ${ }^{33}$. The human participants exercised on a cycle ergometer for $1 \mathrm{~h}$ at $\sim 80 \%$ peak oxygen uptake during each session as described ${ }^{7}$. Skeletal muscle biopsies were taken after an overnight fast prior to the first training session $(n=5)$, and after 10 days, and 14 days $(n=8)$ of exercise training (all biopsies were taken $\sim 16 \mathrm{~h}$ after cessation of the last exercise bout). Biopsies were taken from vastus lateralis under local anesthesia using a biopsy needle, snap frozen in liquid nitrogen, and stored at $-80^{\circ} \mathrm{C}$.

Human participants for acute exercise study. Healthy sedentary male volunteers completed two isocaloric acute exercise bouts at low $\left(40 \% \mathrm{VO}_{2 \text { peak }}\right)$ and high $(80 \%$ $\mathrm{VO}_{2 \text { peak }}$ ) intensity on two occasions separated by at least 1 week in random order $^{40}$. Skeletal muscle biopsies were obtained at baseline, $3 \mathrm{~h}$, and $19 \mathrm{~h}$ after each respective exercise session. A subset of biopsies obtained from five subjects was retrieved for RNA extraction in this study.

\section{Cell cultures}

Primary human skeletal muscle cells. Human satellite cells were isolated from vastus lateralis skeletal muscle biopsies of healthy volunteers as described ${ }^{41}$. The research ethical committee at Karolinska Institutet approved the study protocols and all volunteers provided informed consent. Experimental procedures were conducted according to the Declaration of Helsinki. Myoblasts were grown and differentiated into multinucleated myotubes as described ${ }^{42}$. Cells were propagated in growth media (F12/DMEM, 20\% FBS, and 1\% anti-anti) and when $~ 90 \%$ confluent, cells were differentiated for 4 days with fusion media (DMEM/M199 (4:1), 1\% FBS, 1\% penicillin-streptomycin/amphotericin B (anti-anti), $20 \mathrm{mM}$ HEPES, $0.03 \mu \mathrm{g} \mathrm{mL}^{-1}$ $\mathrm{ZnSO}_{4}, 1.4 \mu \mathrm{g} \mathrm{mL}^{-1}$ vitamin B12, $10 \mu \mathrm{g} \mathrm{mL}^{-1}$ insulin, and $100 \mu \mathrm{g} \mathrm{mL}^{-1}$ apotransferrin). Thereafter, cells were cultured in post-fusion media (DMEM/M199 (4:1), $1 \%$ FBS, $1 \%$ anti-anti, $20 \mathrm{mM}$ HEPES, $0.03 \mu \mathrm{g} \mathrm{mL}^{-1} \mathrm{ZnSO}_{4}$, and $1.4 \mu \mathrm{g} \mathrm{mL}^{-1}$ vitamin $\mathrm{B} 12$ ). Cultures were incubated at $37^{\circ} \mathrm{C}$ in $7.5 \% \mathrm{CO}_{2}$ humidified chambers, and medium was changed every second day during growth and differentiation.
Transfection of human skeletal muscle cells with either Pre-miR ${ }^{\mathrm{Tm}}$ miRNA Precursors $(25 \mathrm{nM})$, mirVana ${ }^{\circledR}$ miRNA inhibitors $(25 \mathrm{nM})$, or Silencer Select siRNAs $(5 \mathrm{nM})$ was performed 6 days after induction of differentiation. Transfections were performed for $5 \mathrm{~h}$ in OptiMEM reduced serum media with Lipofectamine ${ }^{\circledast}$ RNAiMAX. A second transfection was conducted after $48 \mathrm{~h}$ and all cellular assays were performed 2 days after the second transfection. Media, miRNA precursors and inhibitors, siRNAs, and transfection reagents were from Thermo Fisher Scientific and oligonucleotides used are reported in Supplementary Table 5.

Primary mouse skeletal muscle cells. Primary mouse skeletal muscle cells were isolated as described ${ }^{43}$. Hind limb muscles from 2 -week-old C57BL/6J mice were removed and mechanically minced in PBS. Thereafter, enzymatic dissociation of satellite cells was performed with Collagenase and Dispase (Thermo Fisher Scientific, MA) and the suspension was passed through a $70 \mu \mathrm{m}$ cell strainer. Differential plating was performed during the initial passages to enrich for myoblasts. Cells were grown on collagen-coated plates with growth media (DMEM/Ham's F10 (1:1), 20\% FBS, 1\% penicillin-streptomycin/amphotericin B (anti-anti), and $2.5 \mathrm{ng} \mathrm{mL}^{-1} \mathrm{bFGF}$ ). Differentiation was induced after plating $83,300 \mathrm{cells} \mathrm{cm}^{-2}$ and $\sim 12 \mathrm{~h}$ later switching to differentiation media (DMEM, $5 \%$ horse serum, and $1 \%$ anti-anti). Cultures were incubated at $37^{\circ} \mathrm{C}$ in $5 \% \mathrm{CO}_{2}$ humidified chambers, and medium was changed every second day during growth and differentiation.

Cells were differentiated for 2 days and transfected with miRNA Pre-miR ${ }^{\text {ns }}$ miRNA Precursors $(25 \mathrm{nM})$ for $5 \mathrm{~h}$ in OptiMEM reduced serum media with Lipofectamine $^{\circledast}$ RNAiMAX. Two days after a single transfection, final experiments were performed. Media, miRNA precursors, and transfection reagents were from Thermo Fisher Scientific.

C2C12 skeletal muscle cells. Mouse C2C12 myoblasts (ATCC, VA) were propagated in growth media (DMEM, 20\% FBS, and 1\% anti-anti). Cells were seeded $\left(42,000\right.$ cells $\left.\mathrm{cm}^{-2}\right)$ and $\sim 12 \mathrm{~h}$ thereafter the culture medium was changed to differentiation medium (DMEM, $2 \%$ horse serum, and $1 \%$ anti-anti). Cells were incubated at $37^{\circ} \mathrm{C}$ in $5 \% \mathrm{CO}_{2}$ humidified chambers, and medium was changed every second day during growth and differentiation. Cells were differentiated for 3 days and then transfected using a double transfection protocol. Cells were transfected with miRNA Pre-miR ${ }^{m}$ miRNA Precursors $(25 \mathrm{nM})$ for $5 \mathrm{~h}$ in OptiMEM reduced serum media with Lipofectamine ${ }^{\circledR}$ RNAiMAX. For the double transfection protocol, cells were transfected again $48 \mathrm{~h}$ after the first transfection and final experiments were performed 2 days later.

Short RNA sequencing and analysis. Short RNA sequencing of human skeletal muscle was performed using a previously described protocol ${ }^{44}$. Total RNA was extracted from human skeletal muscle biopsies using TRIzol. Small RNA molecules 18-24 nucleotides long were purified using mirVana ${ }^{\mathrm{Tx}}$ miRNA Isolation Kit (Thermo Fisher Scientific) followed by $15 \%$ PAGE/7 M urea. Thereafter, ligation of $3^{\prime}$ - and $5^{\prime}$-adaptors was performed, cDNA was synthetized and amplified, and miRNA abundance was determined by sequencing (Illumina, CA). Short RNA sequencing reads were trimmed using FastX followed by mapping to the hg38 reference genome using Bowtie ${ }^{45}$. miRNA coverages were computed using featureCounts ${ }^{46}$ with miRBase v21 annotations. miRNAs with expression $<100$ reads per million (RPM) in more than three samples were excluded from the differential expression analysis since they are unlikely to be active ${ }^{12}$. Differential expression of miRNAs was determined using the edgeR glmQLFit/glmQLFTest framework $^{47}$ and corrected for multiple testing using the Benjamini-Hochberg correction.

Glucose uptake measurements in cellular models. Glucose uptake was determined in primary human skeletal muscle cells as described ${ }^{42}$. Cells were starved of serum for $4 \mathrm{~h}$ prior to treatment with insulin. Glucose uptake was determined following incubation of cells for $1 \mathrm{~h}$ in absence or presence of insulin, prior to addition of 2-[1,2- $\left.{ }^{3} \mathrm{H}\right]$ deoxy-D-glucose (Moravek Inc., CA) and $10 \mathrm{mM}$ unlabeled 2 -deoxy-D-glucose for $15 \mathrm{~min}$. Cells were homogenized with $0.03 \% \mathrm{SDS}$ and the amount of $2-\left[1,2-{ }^{3} \mathrm{H}\right]$ deoxy-D-glucose in the homogenates was determined by scintillation counting.

Glucose uptake in primary mouse skeletal muscle cells was determined as described $^{48}$. Cells were serum-starved for $4 \mathrm{~h}$ before incubation in the absence or presence of insulin for $20 \mathrm{~min}$. Thereafter, cells were rinsed twice with HEPESbuffered saline (140 mM NaCl, $20 \mathrm{mM} \mathrm{Na-HEPES,} 2.5 \mathrm{mM} \mathrm{MgSO}_{4}, 1 \mathrm{mM} \mathrm{CaCl}_{2}$, $5 \mathrm{mM} \mathrm{KCl}, \mathrm{pH} 7.4$ ) and incubated for $5 \mathrm{~min}$ (with $2-\left[1,2-{ }^{3} \mathrm{H}\right]$ deoxy-D-glucose and $10 \mathrm{mM}$ unlabeled 2-deoxy-D-glucose.

C2C12 myotubes were serum-starved for $4 \mathrm{~h}$ and then incubated in the absence or presence of insulin. Thereafter, glucose uptake was determined for $15 \mathrm{~min}$ in cells incubated with $2-\left[1,2-{ }^{3} \mathrm{H}\right]$ deoxy-D-glucose and $10 \mathrm{mM}$ unlabeled 2-deoxy-Dglucose. Cells were homogenized with $0.03 \%$ SDS and the amount of $2-\left[1,2-{ }^{3} \mathrm{H}\right]$ deoxy-D-glucose in homogenates was determined by scintillation counting.

Normalization of glucose uptake was performed to total protein content (Pierce BCA Protein Assay Kit, Thermo Fisher Scientific) and scintillation counting was determined using a 1414 WinSpectral Liquid Scintillation Counter (Wallac, Perkin-Elmer, MA). All radioisotopes were acquired from Perkin-Elmer if not otherwise stated. 


\section{Glycogen synthesis analysis in primary human and mouse skeletal muscle} cells. Glucose incorporation into glycogen was determined in primary human and mouse skeletal muscle cells as described ${ }^{42}$. Cells were starved of serum for $4 \mathrm{~h}$ prior to treatment with insulin and thereafter incubated for $30 \mathrm{~min}$ in the absence or presence of insulin followed by addition of $\mathrm{D}-\left[\mathrm{U}-{ }^{14} \mathrm{C}\right]$ glucose (Perkin-Elmer) for $90 \mathrm{~min}$. Cells were homogenized, glycogen was precipitated with ethanol at $-20^{\circ} \mathrm{C}$ overnight, and the amount of $\mathrm{D}-\left[\mathrm{U}-{ }^{14} \mathrm{C}\right]$ glucose-containing glycogen was determined by scintillation counting. Normalization of glucose incorporation into glycogen was performed to total protein content (Pierce BCA Protein Assay Kit, Thermo Fisher Scientific) and scintillation counting was determined using a 1414 WinSpectral Liquid Scintillation Counter.

Glucose and palmitate oxidation in cells. Glucose and palmitate oxidation were determined in primary human skeletal muscle cells as described ${ }^{42}$. Glucose oxidation was determined by incubation of cells with $\mathrm{D}-\left[\mathrm{U}-{ }^{14} \mathrm{C}\right]$ glucose in the absence or presence of $1 \mu \mathrm{M}$ FCCP (Sigma-Aldrich). Plates were sealed and incubated for $4 \mathrm{~h}$. Thereafter, media was acidified $(1: 8 \mathrm{vol} 2 \mathrm{M} \mathrm{HCl})$ and the liberated ${ }^{14} \mathrm{CO}_{2}$ was collected for $1 \mathrm{~h}$ in a center well containing $2 \mathrm{M} \mathrm{NaOH}$. The amount of liberated ${ }^{14} \mathrm{CO}_{2}$ was determined by scintillation counting. Palmitate oxidation was determined following pre-treatment of cells with or without $10 \mathrm{nM}$ GW1516 (GW501516, Sigma-Aldrich) for $96 \mathrm{~h}$. Cells were incubated with $25 \mu \mathrm{M}$ non-labeled palmitic acid and $\left[9,10-{ }^{3} \mathrm{H}\right]$ palmitic acid for $6 \mathrm{~h}$. The amount of ${ }^{3} \mathrm{H}_{2} \mathrm{O}$ released into culture media was determined by scintillation counting. Results were normalized to total cellular protein content (Pierce BCA Protein Assay Kit, Thermo Fisher Scientific) and scintillation counting was determined using a 1414 WinSpectral Liquid Scintillation Counter.

Extracellular flux analysis (Seahorse). Mitochondrial function was assessed using Seahorse XF Cell Mito Stress Test Kit (Agilent Technologies, CA). Cells were plated at a density of 30,000 cells per well in Seahorse XF24 Cell Culture Microplates. Differentiation was initiated the following day with addition of fusion media for 4 days and cells were subsequently cultured for 2 days in post fusion media. A double transfection was performed and the assay was performed $48 \mathrm{~h}$ after the second transfection. Cells were incubated in XF Base Medium supplemented with $5 \mathrm{mM}$ glucose, $1 \mathrm{mM}$ pyruvate, and $2 \mathrm{mM}$ L-glutamine. OCR values were obtained at baseline and after addition of $1 \mu \mathrm{M}$ oligomycin, $2 \mu \mathrm{M}$ FCCP, and $0.75 \mu \mathrm{M}$ rotenone $+0.75 \mu \mathrm{M}$ antimycin A. OCR values were normalized to protein content (Pierce BCA Protein Assay Kit, Thermo Fisher Scientific).

Animal experiments. All animal experiments were approved by the Regional Animal Ethical Committee (Stockholm, Sweden) and all work was performed in adherence with relevant guidelines. Fifteen-week-old male C57BL/6J mice were purchased from Charles River Laboratories (Sulzfeld, Germany). Animals were housed under a 12-h light/12-h dark cycle, $24^{\circ} \mathrm{C}$ and $45 \%$ humidity, and received ad libitum access to water and standard rodent chow (Lantmännen, Sweden). Mice were acclimatized for at least 1 week prior to experimental interventions.

Transcardial perfusion. Transcardial perfusion with PBS was performed in mice anesthetized with Avertin (2,2,2-tribromoethanol and tertiary amyl alcohol) until the perfused heart was free of blood. Soleus and extensor digitorum longus (EDL) skeletal muscles were removed and immediately frozen in liquid nitrogen for subsequent analysis.

Electroporation of mouse skeletal muscle. C57Bl/6J male mice were anesthetized with isoflurane and tibialis anterior (TA) muscles injected with hyaluronidase $\left(30 \mu \mathrm{l} ; 1\right.$ unit $\left.\mathrm{mL}^{-1}\right)$. Mice received carprofen $\left(5 \mathrm{mg} \mathrm{kg}^{-1}\right)$ subcutaneously. Mice were then single-housed for $2 \mathrm{~h}$ prior to a second anesthesia with isoflurane. Intramuscular injection with $30 \mu \mathrm{g}$ of a plasmid encoding either pri-miR-19b-3p or a control plasmid (Origene, MD) in the contralateral leg and electroporation was performed by delivering $220 \mathrm{~V} \mathrm{~cm}^{-1}$ as 8 pulses of $20 \mathrm{~ms}$ using an electroporator (ECM 830 Electroporator, BTX, Harvard Bioscience company) ${ }^{49}$. One week after electroporation, mice were fasted for $4 \mathrm{~h}$ and anesthetized with Avertin. Tibialis anterior muscles were removed and instantly frozen in liquid nitrogen for subsequent analysis. Flexor digitorum brevis (FDB) muscles were electroporated with $20 \mu \mathrm{g}$ of a plasmid encoding either pri-miR-19b-3p or a control plasmid on the contralateral foot at $100 \mathrm{~V} \mathrm{~cm}^{-1}$ as 20 pulses of $20 \mathrm{~ms}$ using an electroporator ${ }^{50}$ One week after electroporation FDB muscles were carefully dissected out and glucose uptake was assessed following contraction.

Intramuscular delivery of adenovirus into mouse skeletal muscle. Human Type 5 (dE1/E3) adenoviruses expressing green fluorescent protein (GFP) alone or $m m u-$ miR-19b1 and GFP under independent CMV promoters (Vector Biolabs, Malvern, PA) were injected into FDB muscles of 3-week-old C57Bl6J ( $2 \mu \mathrm{L} / \mathrm{g}$ of mice, $2 \times 1010$ infectious particles) ${ }^{51}$. Seven days post-injection FDB muscles were carefully dissected out and glucose uptake was assessed following contraction.

\section{Glucose uptake in mouse flexor digitorum brevis skeletal muscle in vitro} Glucose uptake in electroporated FDB skeletal muscle was assessed following contraction or insulin-stimulation, as previously described ${ }^{52}$. Incubations were performed at $30^{\circ} \mathrm{C}$ in Krebs-Henseleit bicarbonate (KHB) buffer supplemented with $0.1 \%$ BSA (RIA grade) that were continuously gassed with $95 \% \mathrm{O}_{2}$ and $5 \%$ $\mathrm{CO}_{2}$. Insulin-stimulated glucose uptake was determined following a recovery period of $30 \mathrm{~min}$ and thereafter FDB muscles were incubated in the absence or presence of insulin $(0.36 \mathrm{nM})$ for $30 \mathrm{~min}$ in $\mathrm{KHB}$ containing $5 \mathrm{mM}$ glucose and $15 \mathrm{mM}$ mannitol. Contraction-induced glucose uptake was performed using a myograph system (DMT A/S, Denmark) and recorded and analyzed using Labchart v7 software (ADInstruments, New Zealand). Paired FDB muscles were placed in $\mathrm{KHB}$ containing $5 \mathrm{mM}$ glucose and $15 \mathrm{mM}$ mannitol for a 20 -min-period before being subjected to either electrical stimulation $(100 \mathrm{~Hz}, 0.2 \mathrm{~ms}$ pulse duration, $\sim 20 \mathrm{~V}$, one contraction of $0.2 \mathrm{~s}$ every $2 \mathrm{~s}$ ) or rest condition for $10 \mathrm{~min}$. After contraction or insulin-stimulation, muscles were incubated in KHB supplemented with $20 \mathrm{mM}$ mannitol for $10 \mathrm{~min}$ before incubation in KHB supplemented with $1 \mathrm{mM} 2$-deoxyglucose and $\left[{ }^{3} \mathrm{H}\right] 2$-deoxy-glucose $\left(2.5 \mu \mathrm{Ci} \mathrm{mL}^{-1}\right.$, American Radiolabeled Chemicals, $\mathrm{MO})$ and $19 \mathrm{mM}$ mannitol and $\left[{ }^{14} \mathrm{C}\right]$ mannitol $\left(0.7 \mathrm{mCi} \mathrm{mL}^{-1}\right.$, Moravek Inc., CA) for $20 \mathrm{~min}$. Thereafter, muscles were stripped of connective tissue and snap frozen in liquid nitrogen and later homogenized in homogenization buffer $\left(137 \mathrm{mM} \mathrm{NaCl}, 2.7 \mathrm{mM} \mathrm{KCl}, 1 \mathrm{mM} \mathrm{MgCl}, 0.5 \mathrm{mM} \mathrm{Na}_{3} \mathrm{VO}_{4}, 1 \%\right.$ (vol/vol) Triton X-100, 10\% (vol/vol) glycerol, $20 \mathrm{mM}$ Tris, $10 \mathrm{mM} \mathrm{NaF}, 1 \mathrm{mM}$ EDTA, $1 \mathrm{mM}$ PMSF, and 1\% (vol/vol) Protease Inhibitor Cocktail Set 1 (Merck Millipore, MA)). Glucose uptake was determined by scintillation counting and was normalized to protein content (Pierce BCA Protein Assay Kit, Thermo Fisher Scientific).

RNA isolation and miRNA and gene expression analysis by real-time qPCR. Total RNA from human skeletal muscle biopsies, mouse skeletal muscles and cells were extracted using TRIzol according to manufacturer's instructions (Thermo Fisher Scientific). RNA concentration was determined by spectrophotometry using a Nanodrop ND-1000 (Thermo Fisher Scientific) and equal amounts of RNA were used for cDNA synthesis. cDNA was synthesized using the High Capacity cDNA Reverse Transcription kit with random primers or the TaqMan ${ }^{\mathrm{mi}}$ Advanced miRNA cDNA Synthesis kit (Thermo Fisher Scientific). RT-qPCR was performed using either a ViiA 7 Real-Time PCR 384-well system or a StepOne Plus Real-Time PCR 96-well system (Thermo Fisher Scientific). For determination of relative miRNA abundance, TaqMan Advanced miRNA primers (Supplementary Table 5) and $\mathrm{TaqMan}^{\circledast}$ Fast Advanced Master Mix were used according to manufacturer's recommendation (Thermo Fisher Scientific) and normalization was performed to miR-186-5p expression (Thermo Fisher Scientific recommended reference miRNA). Gene expression was determined using Fast SYBR $^{m}$ Green Master Mix (Thermo Fisher Scientific) and primers used are reported in Supplementary Table 6. Gene expression in human skeletal muscle biopsies and cells was normalized to the geometrical mean of GUSB and TBP expression. For C2C12 cells, primary mouse skeletal muscle cells and mouse TA muscle, gene expression was normalized to $T b p$ expression.

Transcriptomic analysis and identification of miR-19b-3p targets. RNA from human skeletal muscle cells transfected with Pre-miR ${ }^{\mathrm{m} x}$ miRNA Precursors for miR$19 b-3 p$ or Pre-miR ${ }^{\text {ma }}$ miRNA Negative Control was subjected to a transcriptomic analysis. Total RNA (100 ng) was used to generate amplified sense strand DNA followed by fragmentation and biotinylation using the WT Plus Reagent kit (Thermo Fisher Scientific). ssDNA was hybridized to Clariom S gene array chips (Affymetrix, CA). Array chips were washed, stained, and scanned using a Gene Titan instrument (Thermo Fisher Scientific).

Preprocessing of data was performed using Robust Multiarray Average (RMA) using the oligo package ${ }^{53}$ with the $\mathrm{R}$ software ${ }^{54}$. After removal of non-annotated genes, the probe level expression was collapsed to gene level (average method) using the WGCNA package ${ }^{55}$. Differentially expressed genes (DEGs) were determined with the linear models for microarray (limma) package ${ }^{55}$, following overexpression of either miR-19b-3p relative to Negative Control transfected cells. DEGs were determined by a paired test and defined as $p$-value $<0.01$ and at least 1.5 -fold change. Gene ontology (GO) enrichment analysis was performed with DEGs as input using clusterProfiler ${ }^{56}$. The transcriptomic data are available through NCBI's GEO with accession number GSE126187.

To find miR-19b-3p predicted target genes altered by aerobic exercise training, three publically available microarray studies were utilized. Data from GSE35661 compares vastus lateralis biopsies from 24 healthy sedentary male volunteers before and after 6 weeks of aerobic exercise training ${ }^{8}$, data from GSE27536 compares vastus lateralis biopsies from 12 healthy volunteers $(10$ males and 2 females; patients with chronic obstructive pulmonary disease were excluded from analysis) before and after a 8-week aerobic exercise program ${ }^{16}$, and data from GSE9103 compares vastus lateralis biopsies from young volunteers who were either sedentary ( 5 women, 6 men) or exercise-trained ( 5 women, 6 men; self-reported exercise of at least $1 \mathrm{~h}$ of cycling or running 6 days per week over the past 4 years) $)^{15}$. Data was generated from NCBI's GEO depository and analyzed with either GEO2R (GSE9103) or in-house using R with RMA preprocessing (GSE27536 and GSE35661 using paired statistical analysis). Potential aerobic exercise-responsive miR-19b-3p-regulated genes were defined as having (1) altered expression in at least two exercise training studies $(p<0.05)$, (2) similar fold change directionality comparing miRNA overexpression and following exercise training, and (3) being predicted as miR-19b-3p targets using either TargetScan (release 7.1$)^{57}$ or microRNA.org 58 
Protein extraction and western blot analysis. Western blot was utilized for determination of protein abundance and phosphorylation status in skeletal muscle cells and tissue. FDB muscles were frozen immediately following glucose transport experiments. Human skeletal muscle cells were serum-starved for $4 \mathrm{~h}$ and thereafter incubated in the absence or presence of insulin for $10 \mathrm{~min}$. Cells and tissues were lysed in ice-cold homogenization buffer $(137 \mathrm{mM} \mathrm{NaCl}, 2.7 \mathrm{mM}$ $\mathrm{KCl}, 1 \mathrm{mM} \mathrm{MgCl} 2,0.5 \mathrm{mM} \mathrm{Na}_{3} \mathrm{VO}_{4}, 1 \%$ (vol/vol) Triton X-100, 10\% (vol/vol) glycerol, $20 \mathrm{mM}$ Tris, $10 \mathrm{mM} \mathrm{NaF}, 1 \mathrm{mM}$ EDTA, $1 \mathrm{mM}$ PMSF, and 1\% (vol/vol) Protease Inhibitor Cocktail Set 1 (Merck Millipore, MA)). Lysates were rotated at $4^{\circ} \mathrm{C}$ for $30 \mathrm{~min}$ before being subjected to centrifugation at $12,000 \times g$ at $4{ }^{\circ} \mathrm{C}$ for $15 \mathrm{~min}$. Protein concentration was determined by Pierce BCA Protein Assay Kit (Thermo Fisher Scientific) and equal amounts of protein were diluted with Laemmli buffer. Samples were heated at $56^{\circ} \mathrm{C}$ for $15 \mathrm{~min}$ before being separated by SDS-PAGE using Criterion XT Bis-Tris Gels (Bio-Rad, CA). Proteins were transferred to PVDF membranes (Merck Millipore) and Ponceau S staining was used to confirm equal loading and transfer (Sigma-Aldrich, MO). Membranes were blocked in $7.5 \%$ milk in TBST $(10 \mathrm{mM}$ Tris- $\mathrm{HCl}, 100 \mathrm{mM} \mathrm{NaCl}, 0.02 \%$ Tween 20) for $1 \mathrm{~h}$ at RT and thereafter incubated at $4{ }^{\circ} \mathrm{C}$ overnight with primary antibodies (listed in Supplementary Table 7). Membranes were washed with TBST, incubated for $1 \mathrm{~h}$ at RT with appropriate secondary antibodies, washed with TBST, and then visualized by enhanced chemiluminescence (ECL Western Blotting Detection Reagent, GE Healthcare, IL). Protein content was quantified by densitometry (QuantityOne, Bio-Rad). Full scan blots are presented in the Source data file.

Quantification and statistical analysis. Data are presented as mean \pm SEM (if not otherwise stated in Figure Legends). Statistical analysis of RNA sequencing and gene array analysis are described above. Normal distribution of the data was tested with Shapiro-Wilk test. Differences in miRNA and mRNA expression in human biopsies were determined by paired Student's $t$-test or repeated-measures ANOVA followed by Dunnett's test for post hoc pair-wise comparisons or using Friedman's test followed by Dunn's multiple comparison test when appropriate. For cell culture and animal experiments, miRNA and gene expression were analyzed by paired Student's $t$-test and metabolic assays were analyzed using repeated-measures or two-way ANOVA followed by appropriate test for post hoc pair-wise comparisons. Time to 50\% fatigue in FDB skeletal muscle was determined by plotting a logarithmic line to data representing force production over time during the contraction time-course. Pearson correlations between exercise training-induced fold change in miRNA and gene expression was performed following centring of each individuals genes and miRNAs expression to the individuals average expression (defined as FoldExpression $_{\text {Timepoint:n }}=$ Expression $_{\text {Timepoint: }} /$ average $\left(\right.$ Expression $_{\text {timepoint:Baseline }}+$ Expression $_{\text {timepoint:10days }}+$ Expression $\left._{\text {timepoint:14days }}\right)$ ) to reduce variation between subject's baseline expression to study exercise training-specific effects on gene and miRNA expression. Comparisons were considered statistically significant at $p<0.05$ and analyses were performed using GraphPad Prism 8 software (GraphPad Software Inc., CA).

Reporting summary. Further information on research design is available in the Nature Research Reporting Summary linked to this article.

\section{Data availability}

Short RNA-seq data generated in this study have been deposited in the NCBI's GEO database under accession number GSE127187. The GEO accession number for the gene array-generated transcriptomic data reported in this paper is GSE126187. Raw data of all figures and uncropped versions of blots presented in the figures are provided as a Source data file. The NCBI's GEO accession numbers for published microarray data analyzed in this paper are GSE $35661^{8}$, GSE27536 $6^{16}$, and GSE9103 ${ }^{15}$. Source data are provided with this paper.

\section{Code availability}

$\mathrm{R}$ scripts for the main steps of analysis are available from the corresponding author on reasonable request.

Received: 31 March 2019; Accepted: 9 September 2021;

Published online: 12 October 2021

\section{References}

1. Egan, B. \& Zierath, J. R. Exercise metabolism and the molecular regulation of skeletal muscle adaptation. Cell Metab. 17, 162-184 (2013).

2. Rogers, M. A., King, D. S., Hagberg, J. M., Ehsani, A. A. \& Holloszy, J. O. Effect of 10 days of physical inactivity on glucose tolerance in master athletes. J. Appl. Physiol. 68, 1833-1837 (1990).

3. Morrison, D. J., Kowalski, G. M., Grespan, E., Mari, A., Bruce, C. R. \& Wadley, G. D. Measurement of postprandial glucose fluxes in response to acute and chronic endurance exercise in healthy humans. Am. J. Physiol. Endocrinol. Metab. 314, E503-E511 (2018).

4. Devlin, J. T., Hirshman, M., Horton, E. D. \& Horton, E. S. Enhanced peripheral and splanchnic insulin sensitivity in NIDDM men after single bout of exercise. Diabetes 36, 434-439 (1987).

5. Booth, F. W., Roberts, C. K. \& Laye, M. J. Lack of exercise is a major cause of chronic diseases. Compr. Physiol. 2, 1143-1211 (2012).

6. Colberg, S. R. et al. Physical activity/exercise and diabetes: a position statement of the american diabetes association. Diabetes Care 39, 2065-2079 (2016).

7. Egan, B., O'Connor, P. L., Zierath, J. R. \& O'Gorman, D. J. Time course analysis reveals gene-specific transcript and protein kinetics of adaptation to short-term aerobic exercise training in human skeletal muscle. PLOS ONE 8 e74098 (2013)

8. Keller, P. et al. A transcriptional map of the impact of endurance exercise training on skeletal muscle phenotype. J. Appl Physiol. 110, 46-59 (2011).

9. Ambros, V. The functions of animal microRNAs. Nature 431, 350-355 (2004).

10. Nielsen, S. et al. Muscle specific microRNAs are regulated by endurance exercise in human skeletal muscle. J. Physiol. 588, 4029-4037 (2010).

11. Russell, A. P. et al. Regulation of miRNAs in human skeletal muscle following acute endurance exercise and short-term endurance training. J. Physiol. 591, 4637-4653 (2013).

12. Mullokandov, G. et al. High-throughput assessment of microRNA activity and function using microRNA sensor and decoy libraries. Nat. Methods $\mathbf{9}$, 840-846 (2012).

13. Al-Khalili, L. et al. Insulin action in cultured human skeletal muscle cells during differentiation: assessment of cell surface GLUT4 and GLUT1 content. Cell Mol. Life Sci. 60, 991-998 (2003).

14. Vichaiwong, K. et al. Contraction regulates site-specific phosphorylation of TBC1D1 in skeletal muscle. Biochem. J. 431, 311-320 (2010).

15. Lanza, I. R. et al. Endurance exercise as a countermeasure for aging. Diabetes 57, 2933-2942 (2008).

16. Turan, N. et al. A systems biology approach identifies molecular networks defining skeletal muscle abnormalities in chronic obstructive pulmonary disease. PLoS Comput. Biol. 7, e1002129 (2011).

17. Davidsen, P. K. et al. High responders to resistance exercise training demonstrate differential regulation of skeletal muscle microRNA expression. J. Appl. Physiol. 110, 309-317 (2011).

18. Patrick, D. M. et al. Defective erythroid differentiation in miR-451 mutant mice mediated by 14-3-3zeta. Genes Dev. 24, 1614-1619 (2010).

19. de Gonzalo-Calvo, D. et al. Circulating inflammatory miRNA signature in response to different doses of aerobic exercise. J. Appl. Physiol. 119, 124-134 (2015).

20. Nielsen, S. et al. The miRNA plasma signature in response to acute aerobic exercise and endurance training. PLoS ONE 9, e87308 (2014).

21. Chen, X., Liang, H., Zhang, J., Zen, K. \& Zhang, C. Y. Secreted microRNAs: a new form of intercellular communication. Trends Cell Biol. 22, 125-132 (2012).

22. Safdar, A., Abadi, A., Akhtar, M., Hettinga, B. P. \& Tarnopolsky, M. A. miRNA in the regulation of skeletal muscle adaptation to acute endurance exercise in C57Bl/6J male mice. PLoS ONE 4, e5610 (2009).

23. McCarthy, J. J., Esser, K. A., Peterson, C. A. \& Dupont-Versteegden, E. E. Evidence of MyomiR network regulation of beta-myosin heavy chain gene expression during skeletal muscle atrophy. Physiol. Genomics 39, 219-226 (2009).

24. Li, H. et al. circFGFR4 promotes differentiation of myoblasts via binding miR107 to relieve its inhibition of Wnt3a. Mol. Ther. Nucleic Acids 11, 272-283 (2018).

25. Barres, R. et al. Acute exercise remodels promoter methylation in human skeletal muscle. Cell Metab. 15, 405-411 (2012).

26. McLean, C. S. et al. Gene and microRNA expression responses to exercise; relationship with insulin sensitivity. PLoS ONE 10, e0127089-e0127089 (2015).

27. Mogilyansky, E. \& Rigoutsos, I. The miR-17/92 cluster: a comprehensive update on its genomics, genetics, functions and increasingly important and numerous roles in health and disease. Cell Death Differ. 20, 1603-1614 (2013).

28. Zhang, $\mathrm{H}$. et al. Acetylation of AGO2 promotes cancer progression by increasing oncogenic miR-19b biogenesis. Oncogene 38, 1410-1431 (2019).

29. Ploug, T. et al. Effect of endurance training on glucose transport capacity and glucose transporter expression in rat skeletal muscle. Am. J. Physiol. 259, E778-786 (1990).

30. Treebak, J. T. et al. Acute exercise and physiological insulin induce distinct phosphorylation signatures on TBC1D1 and TBC1D4 proteins in human skeletal muscle. J. Physiol. 592, 351-375 (2014).

31. Perseghin, G. et al. Increased glucose transport-phosphorylation and muscle glycogen synthesis after exercise training in insulin-resistant subjects. N. Engl. J. Med. 335, 1357-1362 (1996). 
32. Hawley, J. A. \& Lessard, S. J. Exercise training-induced improvements in insulin action. Acta Physiol. 192, 127-135 (2008).

33. Egan, B. et al. 2-D DIGE analysis of the mitochondrial proteome from human skeletal muscle reveals time course-dependent remodelling in response to 14 consecutive days of endurance exercise training. Proteomics 11, 1413-1428 (2011).

34. Timmons, J. A. et al. Modulation of extracellular matrix genes reflects the magnitude of physiological adaptation to aerobic exercise training in humans. BMC Biol. 3, 19 (2005).

35. Garg, K. \& Boppart, M. D. Influence of exercise and aging on extracellular matrix composition in the skeletal muscle stem cell niche. J. Appl Physiol. 121, 1053-1058 (2016).

36. Tarpey, M. D. et al. Characterization and utilization of the flexor digitorum brevis for assessing skeletal muscle function. Skelet. Muscle 8, 14 (2018).

37. Ashraf, U. et al. MicroRNA-19b-3p modulates japanese encephalitis virusmediated inflammation via targeting RNF11. J. Virol. 90, 4780-4795 (2016).

38. Shembade, N., Parvatiyar, K., Harhaj, N. S. \& Harhaj, E. W. The ubiquitinediting enzyme A20 requires RNF11 to downregulate NF-kappaB signalling. EMBO J. 28, 513-522 (2009)

39. Azmi, P. \& Seth, A. RNF11 is a multifunctional modulator of growth factor receptor signalling and transcriptional regulation. Eur. J. Cancer 41, 2549-2560 (2005).

40. Egan, B. et al. Exercise intensity-dependent regulation of peroxisome proliferator-activated receptor $\gamma$ coactivator- $1 \alpha$ mRNA abundance is associated with differential activation of upstream signalling kinases in human skeletal muscle. J. Physiol. 588, 1779-1790 (2010).

41. Al-Khalili, L., Kramer, D., Wretenberg, P. \& Krook, A. Human skeletal muscle cell differentiation is associated with changes in myogenic markers and enhanced insulin-mediated MAPK and PKB phosphorylation. Acta Physiol. Scand. 180, 395-403 (2004).

42. Massart, J. et al. Altered miR-29 expression in type 2 diabetes influences glucose and lipid metabolism in skeletal muscle. Diabetes 66, 1807-1818 (2017).

43. Rando, T. A. \& Blau, H. M. Primary mouse myoblast purification, characterization, and transplantation for cell-mediated gene therapy. J. Cell Biol. 125, 1275-1287 (1994).

44. Gu, W. et al. Distinct argonaute-mediated 22G-RNA pathways direct genome surveillance in the C. elegans germline. Mol. Cell 36, 231-244 (2009).

45. Langmead, B. \& Salzberg, S. L. Fast gapped-read alignment with Bowtie 2. Nat. Methods 9, 357-359 (2012).

46. Liao, Y., Smyth, G. K. \& Shi, W. featureCounts: an efficient general purpose program for assigning sequence reads to genomic features. Bioinformatics $\mathbf{3 0}$, 923-930 (2014).

47. Robinson, M. D., McCarthy, D. J. \& Smyth, G. K. edgeR: a Bioconductor package for differential expression analysis of digital gene expression data. Bioinformatics 26, 139-140 (2010).

48. Sweeney, G., Somwar, R., Ramlal, T., Volchuk, A., Ueyama, A. \& Klip, A. An inhibitor of p38 mitogen-activated protein kinase prevents insulin-stimulated glucose transport but not glucose transporter translocation in 3T3-L1 adipocytes and L6 myotubes. J. Biol. Chem. 274, 10071-10078 (1999).

49. Kulkarni, S. S., Karlsson, H. K., Szekeres, F., Chibalin, A. V., Krook, A. \& Zierath, J. R. Suppression of 5'-nucleotidase enzymes promotes AMPactivated protein kinase (AMPK) phosphorylation and metabolism in human and mouse skeletal muscle. J. Biol. Chem. 286, 34567-34574 (2011).

50. DiFranco, M., Quinonez, M., Capote, J. \& Vergara, J. DNA transfection of mammalian skeletal muscles using in vivo electroporation. J. Vis. Exp. 32, e1520 (2009)

51. Ferreira, D. M. S. et al. LIM and cysteine-rich domains 1 (LMCD1) regulates skeletal muscle hypertrophy, calcium handling, and force. Skelet. Muscle 9, 26 (2019).

52. Szekeres, F. et al. The Rab-GTPase-activating protein TBC1D1 regulates skeletal muscle glucose metabolism. Am. J. Physiol. Endocrinol. Metab. 303, E524-533 (2012)

53. Carvalho, B. S. \& Irizarry, R. A. A framework for oligonucleotide microarray preprocessing. Bioinformatics 26, 2363-2367 (2010).

54. R Core Team. R: A Language and Environment for Statistical Computing (2013).

55. Langfelder, P. \& Horvath, S. WGCNA: an R package for weighted correlation network analysis. BMC Bioinformatics 9, 559 (2008).
56. Yu, G., Wang, L. G., Han, Y. \& He, Q. Y. clusterProfiler: an R package for comparing biological themes among gene clusters. OMICS 16, 284-287 (2012).

57. Agarwal, V., Bell, G. W., Nam, J. W. \& Bartel, D. P. Predicting effective microRNA target sites in mammalian mRNAs. Elife 4, e05005 (2015).

58. Betel, D., Wilson, M., Gabow, A., Marks, D. S. \& Sander, C. The microRNA.org resource: targets and expression. Nucleic Acids Res. 36, D149-153 (2008)

\section{Acknowledgements}

This work was supported by grant funding from the Swedish Diabetes Foundation, the Novo Nordisk Foundation, Swedish Research Council for Sport Science, the Swedish Research Council, and the European Research Council Ideas program (ICEBERG, ERC2008-AdG233285). We thank the Bioinformatics and Expression Analysis (BEA) core facility at Novum, which is supported by the board of research at Karolinska Institutet and the research committee at Karolinska University Hospital. We also thank Ann-Marie Pettersson for technical assistance and Associate Prof. Christopher Cederroth, Karolinska Institutet, for guidance with cardiac perfusion experiments

\section{Author contributions}

J.M., R.J.O.S., B.E., J.R.Z., and A.K. conceived and designed the research; R.J.O.S., B.E. C.G, W.G., R.B., and D.J.O.G. designed the training study and analyzed RNA sequencing data; J.M., R.J.O.S., B.E., W.G., M.K., D.M.S.F., and M.L. performed experiments; J.M. and R.J.O.S. analyzed data and J.M., R.J.O.S, J.L.R., and A.K. interpreted results of experiments; J.M. and R.J.O.S. prepared figures and tables, and drafted the manuscript; J.M., R.J.O.S., J.R.Z., and A.K. finalized the manuscript.

\section{Funding}

Open access funding provided by Karolinska Institutet.

\section{Competing interests}

The authors declare no competing interests.

\section{Additional information}

Supplementary information The online version contains supplementary material available at https://doi.org/10.1038/s41467-021-26095-0.

Correspondence and requests for materials should be addressed to Anna Krook.

Peer review information Nature Communications thanks Nathan LeBrasseur and the other, anonymous, reviewer(s) for their contribution to the peer review of this work. Peer reviewer reports are available.

Reprints and permission information is available at http://www.nature.com/reprints

Publisher's note Springer Nature remains neutral with regard to jurisdictional claims in published maps and institutional affiliations.

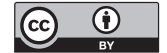

Open Access This article is licensed under a Creative Commons Attribution 4.0 International License, which permits use, sharing, adaptation, distribution and reproduction in any medium or format, as long as you give appropriate credit to the original author(s) and the source, provide a link to the Creative Commons license, and indicate if changes were made. The images or other third party material in this article are included in the article's Creative Commons license, unless indicated otherwise in a credit line to the material. If material is not included in the article's Creative Commons license and your intended use is not permitted by statutory regulation or exceeds the permitted use, you will need to obtain permission directly from the copyright holder. To view a copy of this license, visit http://creativecommons.org/ licenses/by/4.0/.

(c) The Author(s) 2021 